Revised Version

ZU-TH 30/96

TTP96-54

June 1997

\title{
Resonance Contributions to the Electromagnetic Low Energy Constants of Chiral Perturbation Theory
}

\author{
Robert Baur \\ Institut für Theoretische Physik, Universität Zürich \\ CH-8057 Zürich, Switzerland \\ Res Urech \\ Institut für Theoretische Teilchenphysik, Universität Karlsruhe \\ D-76128 Karlsruhe, Germany
}

\begin{abstract}
The effective chiral Lagrangian of the strong and electromagnetic interactions of the pseudoscalar mesons at low energies depends on a set of low energy constants. We determine the contributions to the electromagnetic coupling constants at order $O\left(e^{2} p^{2}\right)$, which arise from resonances within a photon loop. We give some implications of our results, in particular we discuss in detail the effects on the corrections to Dashen's theorem.
\end{abstract}

PACS number(s): 12.39.Fe, 13.40.Dk, 13.40.Ks, 14.40.Aq

${ }^{*}$ e-mail: rbaur@physik.unizh.ch

†e-mail: ru@ttpux2.physik.uni-karlsruhe.de 


\section{Introduction}

At low energies the strong and electromagnetic interactions of the lightest pseudoscalar mesons can be described by an effective field theory, called chiral perturbation theory (CHPT) [1, 2, 3, 4. CHPT provides a systematic expansion in the external momenta $p$, in the light quark masses $m_{q}$ and, in the electromagnetic sector, in the electromagnetic coupling $e$. It is a nonrenormalizable field theory, but it is renormalized order by order in the loop expansion. The effective chiral Lagrangian depends on a number of low energy constants that absorb the ultraviolet divergences generated by the loops. The renormalized couplings are not fixed by chiral symmetry. They are in principle calculable from the underlying dynamics of QCD and QED in terms of the renormalization group invariant scale $\Lambda$ and the heavy quark masses $\left(m_{c}, m_{b}, \ldots\right)$. In practice, however, they are extracted from experimental data, which works to a large extent in the strong sector but hardly in the electromagnetic sector [5].

Complementary to experimental data on may use model assumptions as an input. In the phenomenological picture, the low energy constants get contributions from different sources, such as mesonic resonances, other hadronic states, and even short distance effects. In [6] the authors separated the low energy constants of the strongly interacting sector at next-to-leading order in a part determined by resonance exchange, and a non-resonant remainder. It was found that at the scale point $\mu=M_{\rho}$ the resonance contribution nearly saturates the experimentally known low energy parameters of the strong interaction sector.

In the electromagnetic sector, resonance exchange at tree level cannot be applied because resonances do not contribute to the low energy electromagnetic coupling constants in the absence of photons. Considering the electromagnetic mass shift of the pion in the chiral limit due to photon and resonance exchange at the oneloop level [7], the resonant contribution to the coupling constant $C$ at order $O\left(e^{2}\right)$ was determined in [6] (see also [8]). The occurring divergence was absorbed in the corresponding electromagnetic counterterm Lagrangian associated with the coupling constant $\hat{C}$. It was found that the loop contribution is numerically very close to the experimental value and the authors thus concluded that resonance saturation holds for the leading order electromagnetic coupling constant $C$.

The purpose of this article is to extend the above method to the couplings of the order $O\left(e^{2} p^{2}\right)$. The effective electromagnetic Lagrangian has been given in its general form at leading order in [6] and at next-to-leading order in [9, 10, 11]. In the latter case we have 14 independent operators associated with the coupling constants $K_{i}(i=1 \ldots 14)$. Considering the contributions to the masses, to the scattering amplitudes, and to the matrix elements with external currents (see Section 5) arising from resonances within a photon loop, the resonant contributions to the coupling constants are evaluated. The divergences generated by the loops must be absorbed

in a counterterm Lagrangian with the corresponding coupling constants $\hat{K}_{i}$ [12]. Afterwards we extract the finite contributions from the resonances to the $K_{i}$. 
Since our knowledge on the coupling constants of the electromagnetic sector is still poor, i.e. in general they cannot be related to experimental data, it is not possible to test whether resonance saturation holds in the electromagnetic sector. We are aware of two possible checks. (i) On the one hand we can classify the operators at order $O\left(e^{2} p^{2}\right)$ due to their behaviour in the large $N_{C}$ limit [13] (see Appendix C) and compare them to the numerical result for the couplings $K_{i}$ found in the resonance approach. In the four cases where a suppression by $1 / N_{C}$ occurs we find indeed that the contributions from the resonances vanish, e.g. one of the results is $K_{8}^{R}=0$, where the superscript $R$ indicates resonance contributions. ( $i i)$ On the other hand, exactly this coupling has been estimated in [9] to be $K_{8}^{r}\left(M_{\rho}\right)=-(4.0 \pm 1.7) \times 10^{-3}$, where the superscript $r$ stands for the renormalized coefficient in the Lagrangian $\mathcal{L}_{4}^{Q}$ and the scale point is chosen to $\mu=M_{\rho}$. Obviously, the two results do not coincide. So the only possible hint for a resonance saturation for the $K_{i}$ is found in $(i)$.

We apply our results to different $S$-matrix elements given in the literature (see Section 6), e.g. we discuss the consequences for the corrections to Dashen's theorem [14, 15] at order $O\left(e^{2} m_{q}\right)$. There we find a strong cancellation among the contributions leading to the result that the uncertainties are of the same size (or even larger) than the central value itself.

This article is organized as follows: In Section 2 the Lagrangians of chiral perturbation theory up to the orders $O\left(p^{4}\right)$ and $O\left(e^{2} p^{2}\right)$, respectively, are briefly presented. In Section 3 the linear couplings of the pseudoscalar mesons to the resonances and the relations used among the parameters in the resonance sector are given. Then the general procedure in order to determine the resonance contributions to the low energy coupling constants is described. In Section 5 we present the evaluation of the resonant contributions to the low energy coupling constants $K_{i}$, including an extended discussion of the results. The applications of the numerical results to $S$-matrix elements are given in Section 6 and in the last Section a summary is presented. In Appendix $\mathrm{A}$ we discuss the heavy mass expansion, that we have used in the calculation of the resonance-photon loops at order $O\left(e^{2} p^{2}\right)$. In Appendix $\mathbb{B}$ we list in detail the contributions to the $K_{i}^{R}$ separated for each type of resonances and in Appendix 9 the large $N_{C}$ behaviour of the operators in the Lagrangian $\mathcal{L}_{4}^{Q}$ is deduced.

For comprehensive reviews on CHPT we refer the reader to [ 4 ], a review on the determination of low energy couplings may be found in [5].

\section{Chiral Lagrangian and Low Energy Couplings}

The chiral Lagrangian can be expanded in derivatives of the Goldstone fields and in the masses of the three light quarks. The power counting is established in the following way: The Goldstone fields are of order $O\left(p^{0}\right)$, a derivative $\partial_{\mu}$, the vector and axial vector currents $v_{\mu}, a_{\mu}$ count as quantities of $O(p)$ and the scalar (incorporating the masses) and pseudoscalar currents $s, p$ are of order $O\left(p^{2}\right)$. The effective 
Lagrangian starts at $O\left(p^{2}\right)$, and is denoted by $\mathcal{L}_{2}^{Q}$. It is the non-linear $\sigma$-model Lagrangian coupled to external fields, respects chiral symmetry $S U(3)_{R} \times S U(3)_{L}$, and is invariant under $P$ and $C$ transformations [2]. The Lagrangian $\mathcal{L}_{2}^{Q}$ including electromagnetic interactions can be divided in three parts, namely [6]

$$
\mathcal{L}_{2}^{Q}=\mathcal{L}_{\text {kin }}^{\gamma}+\mathcal{L}_{2}+\mathcal{L}^{C}
$$

where

$$
\begin{aligned}
\mathcal{L}_{k i n}^{\gamma} & =-\frac{1}{4} F_{\mu \nu} F^{\mu \nu}-\frac{\lambda}{2}\left(\partial_{\mu} A^{\mu}\right)^{2} \\
\mathcal{L}_{2} & =\frac{F_{0}^{2}}{4}\left\langle d_{\mu} U d^{\mu} U^{\dagger}\right\rangle+\frac{F_{0}^{2}}{4}\left\langle\chi^{\dagger} U+U^{\dagger} \chi\right\rangle \\
\mathcal{L}^{C} & =C\left\langle Q U Q U^{\dagger}\right\rangle .
\end{aligned}
$$

Here, $F_{\mu \nu}$ is the field strength tensor of the photon field $A_{\mu}$. The parameter $\lambda$ is the gauge fixing parameter, which is set to $\lambda=1$ henceforth. The pseudoscalar meson fields $\phi_{a}$ are contained in the usual way in the matrix $U=\exp \left(i / F_{0} \cdot \sum_{a} \lambda_{a} \phi_{a}\right)$, and the field $\chi$ incorporates the scalar and pseudoscalar currents $s$ and $p$ respectively, $\chi=2 B_{0}(s+i p)$. The quark mass matrix is contained in $s$ (i.e. $\left.s=\mathcal{M}+\cdots\right)$, and we work in the isospin limit $m_{u}=m_{d}=\hat{m}$, i.e. $\mathcal{M}=\operatorname{diag}\left(\hat{m}, \hat{m}, m_{s}\right)$. The covariant derivative $d_{\mu} U$ defines the coupling of the pseudoscalar mesons to the photon field $A_{\mu}$, the external vector and axial vector currents $v_{\mu}$ and $a_{\mu}$ respectively,

$$
d_{\mu} U=\partial_{\mu} U-i\left(v_{\mu}+Q A_{\mu}+a_{\mu}\right) U+i U\left(v_{\mu}+Q A_{\mu}-a_{\mu}\right)
$$

where the charge matrix $Q$ is given by $Q=e \operatorname{diag}(2 / 3,-1 / 3,-1 / 3) . F_{0}$ corresponds to the pion decay constant $F_{\pi}$ in the chiral limit and $B_{0}$ is related to the quark condensate for $m_{q} \rightarrow 0$.

In the chiral limit the masses of the Goldstone bosons are of purely electromagnetic nature, determined by the operator proportional to $C$,

$$
C\left\langle Q U Q U^{\dagger}\right\rangle=-\frac{2 e^{2} C}{F_{0}^{2}}\left(\pi^{+} \pi^{-}+K^{+} K^{-}\right)+O\left(\phi^{4}\right) .
$$

The masses of the charged particles receive an overall shift, whereas the masses of the neutral fields remain zero, in agreement with Dashen's theorem 14.

In order to formally maintain a consistent chiral counting, it is convenient to set 9

$$
e \sim O(p), \quad A_{\mu} \sim O(1)
$$

such that $\mathcal{L}_{2}^{Q}$ is of the order $O\left(p^{2}\right)$.

At next-to-leading order the most general chiral invariant, $\mathrm{P}$ and $\mathrm{C}$ symmetric Lagrangian $\mathcal{L}_{4}^{Q}$ at order $O\left(p^{4}\right)$ has been given in [2] for the strong interaction (associated with the couplings $L_{i}$ and $H_{i}$ ) and in [9, 10, 11] for the electromagnetic 
interaction (couplings $K_{i}$ ),

$$
\begin{aligned}
\mathcal{L}_{4}^{Q}= & L_{1}\left\langle d^{\mu} U^{\dagger} d_{\mu} U\right\rangle^{2}+L_{2}\left\langle d^{\mu} U^{\dagger} d^{\nu} U\right\rangle\left\langle d_{\mu} U^{\dagger} d_{\nu} U\right\rangle \\
& +L_{3}\left\langle d^{\mu} U^{\dagger} d_{\mu} U d^{\nu} U^{\dagger} d_{\nu} U\right\rangle+L_{4}\left\langle d^{\mu} U^{\dagger} d_{\mu} U\right\rangle\left\langle\chi^{\dagger} U+U^{\dagger} \chi\right\rangle \\
& +L_{5}\left\langle d^{\mu} U^{\dagger} d_{\mu} U\left(\chi^{\dagger} U+U^{\dagger} \chi\right)\right\rangle+L_{6}\left\langle\chi^{\dagger} U+U^{\dagger} \chi\right\rangle^{2}+L_{7}\left\langle\chi^{\dagger} U-U^{\dagger} \chi\right\rangle^{2} \\
& +L_{8}\left\langle\chi^{\dagger} U \chi^{\dagger} U+\chi U^{\dagger} \chi U^{\dagger}\right\rangle-i L_{9}\left\langle d^{\mu} U d^{\nu} U^{\dagger} F_{R \mu \nu}+d^{\mu} U^{\dagger} d^{\nu} U F_{L \mu \nu}\right\rangle \\
& +L_{10}\left\langle U^{\dagger} F_{R}^{\mu \nu} U F_{L \mu \nu}\right\rangle+H_{1}\left\langle F_{R}^{\mu \nu} F_{R \mu \nu}+F_{L}^{\mu \nu} F_{L \mu \nu}\right\rangle+H_{2}\left\langle\chi^{\dagger} \chi\right\rangle \\
& +K_{1} F_{0}^{2}\left\langle d^{\mu} U^{\dagger} d_{\mu} U\right\rangle\left\langle Q^{2}\right\rangle+K_{2} F_{0}^{2}\left\langle d^{\mu} U^{\dagger} d_{\mu} U\right\rangle\left\langle Q U Q U^{\dagger}\right\rangle \\
& +K_{3} F_{0}^{2}\left(\left\langle d^{\mu} U^{\dagger} Q U\right\rangle\left\langle d_{\mu} U^{\dagger} Q U\right\rangle+\left\langle d^{\mu} U Q U^{\dagger}\right\rangle\left\langle d_{\mu} U Q U^{\dagger}\right\rangle\right) \\
& +K_{4} F_{0}^{2}\left\langle d^{\mu} U^{\dagger} Q U\right\rangle\left\langle d_{\mu} U Q U^{\dagger}\right\rangle+K_{5} F_{0}^{2}\left\langle\left\{d^{\mu} U^{\dagger}, d_{\mu} U\right\} Q^{2}\right\rangle \\
& +K_{6} F_{0}^{2}\left\langle d^{\mu} U^{\dagger} d_{\mu} U Q U^{\dagger} Q U+d^{\mu} U d_{\mu} U^{\dagger} Q U Q U^{\dagger}\right\rangle \\
& +K_{7} F_{0}^{2}\left\langle\chi^{\dagger} U+U^{\dagger} \chi\right\rangle\left\langle Q^{2}\right\rangle+K_{8} F_{0}^{2}\left\langle\chi^{\dagger} U+U^{\dagger} \chi\right\rangle\left\langle Q U Q U^{\dagger}\right\rangle \\
& +K_{9} F_{0}^{2}\left\langle\left(\chi^{\dagger} U+U^{\dagger} \chi\right) Q^{2}+\left(\chi U^{\dagger}+U \chi^{\dagger}\right) Q^{2}\right\rangle \\
& +K_{10} F_{0}^{2}\left\langle\left(\chi^{\dagger} U+U^{\dagger} \chi\right) Q U^{\dagger} Q U+\left(\chi U^{\dagger}+U \chi^{\dagger}\right) Q U Q U^{\dagger}\right\rangle \\
& +K_{11} F_{0}^{2}\left\langle\left(\chi^{\dagger} U-U^{\dagger} \chi\right) Q U^{\dagger} Q U+\left(\chi U^{\dagger}-U^{\dagger}\right) Q U Q U^{\dagger}\right\rangle \\
& +K_{12} F_{0}^{2}\left\langle d_{\mu} U^{\dagger}\left[c_{R}^{\mu} Q, Q\right] U+d_{\mu} U\left[c_{L}^{\mu} Q, Q\right] U^{\dagger}\right\rangle \\
& +K_{13} F_{0}^{2}\left\langle c_{R}^{\mu} Q U c_{L} Q U^{\dagger}\right\rangle+K_{14} F_{0}^{2}\left\langle c_{R}^{\mu} Q c_{R \mu} Q+c_{L}^{\mu} Q c_{L \mu} Q\right\rangle \\
& +K_{15} F_{0}^{4}\left\langle Q U Q U^{\dagger}\right\rangle^{2}+K_{16} F_{0}^{4}\left\langle Q U Q U^{\dagger}\right\rangle\left\langle Q^{2}\right\rangle+K_{17} F_{0}^{4}\left\langle Q^{2}\right\rangle^{2}
\end{aligned}
$$

where

$$
\begin{aligned}
F_{R, L}^{\mu} & =v^{\mu}+Q A^{\mu} \pm a^{\mu} \\
F_{R, L}^{\mu \nu} & =\partial^{\mu} F_{R, L}^{\nu}-\partial^{\nu} F_{R, L}^{\mu}-i\left[F_{R, L}^{\mu}, F_{R, L}^{\nu}\right] \\
c_{R, L}^{\mu} & =i\left[Q, F_{R, L}^{\mu}\right] .
\end{aligned}
$$

$L_{1} \ldots L_{10}, H_{1}, H_{2}$ and $K_{1} \ldots K_{17}$ are real low energy constants, which are independent of the Goldstone bosons masses and which parameterize all the underlying physics (including e.g. the resonances). The coupling constants $H_{1}, H_{2}, K_{14}$ and $K_{17}$ have no physical significance and are needed for renormalization only. The constants $K_{1}, K_{7}$ and $K_{16}$ are electromagnetic corrections to $F_{0}, B_{0}$, and $C$ respectively. Considering $n$-point functions that involve vector and axial vector currents only, the coupling constant $K_{9}$ can be absorbed in the quark mass matrix,

$$
\mathcal{M} \longrightarrow \mathcal{M}^{Q}=\mathcal{M}\left(\mathbf{1}+8 K_{9} Q^{2}\right)
$$

Note that this substitution can be performed in particular in the corrections to the meson masses. The operators associated with the couplings $K_{15}, K_{16}$ and $K_{17}$ are of the order $O\left(e^{4}\right)$ and are not considered in the following. 


\section{Resonances in the Chiral Lagrangian}

In order to describe the chiral couplings of the resonances to the pseudoscalar Goldstone bosons, we restrict ourselves to the leading order Lagrangian that is linear in the resonance fields. This Lagrangian contains the kinetic term with a covariant derivative and the linear couplings of resonances to the Goldstone bosons and to the external currents in such a way, that all the terms involved are of order $O\left(p^{2}\right)$ [6]. We do not include couplings bilinear in the resonances, (for a systematic treatment in the heavy meson formalism see [19]), nor consider terms linear in the resonances at higher orders in $p^{2}$. Explicitly, the interaction Lagrangian has the form [6]

$$
\begin{aligned}
\mathcal{L}_{2}^{V} & =\frac{F_{V}}{2 \sqrt{2}}\left\langle V_{\mu \nu} f_{+}^{\mu \nu}\right\rangle+\frac{i G_{V}}{2 \sqrt{2}}\left\langle V_{\mu \nu}\left[u^{\mu}, u^{\nu}\right]\right\rangle \\
\mathcal{L}_{2}^{A} & =\frac{F_{A}}{2 \sqrt{2}}\left\langle A_{\mu \nu} f_{-}^{\mu \nu}\right\rangle \\
\mathcal{L}_{2}^{S} & =c_{d}\left\langle S u_{\mu} u^{\mu}\right\rangle+c_{m}\left\langle S \chi_{+}\right\rangle+\tilde{c}_{d} S_{1}\left\langle u_{\mu} u^{\mu}\right\rangle+\tilde{c}_{m} S_{1}\left\langle\chi_{+}\right\rangle,
\end{aligned}
$$

where

$$
\begin{aligned}
f_{ \pm}^{\mu \nu} & =u F_{L}^{\mu \nu} u^{\dagger} \pm u^{\dagger} F_{R}^{\mu \nu} u \\
\chi_{+} & =u^{\dagger} \chi u^{\dagger}+u \chi^{\dagger} u \\
u_{\mu} & =i u^{\dagger} d_{\mu} U u^{\dagger}=u_{\mu}^{\dagger} \\
U & =u^{2} .
\end{aligned}
$$

For the description of the vector $(V)$ and axial vector $(A)$ resonances the antisymmetric tensor notation is used. At leading order, for $V$ and $A$ only the octets couple to the Goldstone bosons whereas for the scalar resonances both the octet $(S)$ and the singlet $\left(S_{1}\right)$ contribute. There exist also similar couplings for the excited pseudoscalar octet $(P)$ and the singlet $\left(P_{1}\right)$, but since they do not generate electromagnetic contributions at the order $O\left(e^{2} p^{2}\right)$ we have omitted the Lagrangian $\mathcal{L}_{2}^{P}$ in Eq.(9). The coupling constants occurring in (9) will be discussed below.

In the kinetic Lagrangian a covariant derivative acts on the octet fields,

$$
\begin{aligned}
\mathcal{L}_{\text {kin }}^{V, A} & =-\frac{1}{2}\left\langle\nabla^{\mu} R_{\mu \nu} \nabla_{\sigma} R^{\sigma \nu}-\frac{1}{2} M_{R}^{2} R_{\mu \nu} R^{\mu \nu}\right\rangle \\
\mathcal{L}_{\text {kin }}^{S} & =\frac{1}{2}\left\langle\nabla^{\mu} S \nabla_{\mu} S-M_{S}^{2} R^{2}\right\rangle+\frac{1}{2}\left(\partial^{\mu} S_{1} \partial_{\mu} S_{1}-M_{S_{1}}^{2} S_{1}^{2}\right) \\
\nabla^{\mu} R_{(\mu \nu)} & =\partial^{\mu} R_{(\mu \nu)}+\left[\Gamma^{\mu}, R_{(\mu \nu)}\right] \\
\Gamma^{\mu} & =\frac{1}{2}\left[u^{\dagger}\left(\partial^{\mu}-i F_{R}^{\mu}\right) u+u\left(\partial^{\mu}-i F_{L}^{\mu}\right) u^{\dagger}\right]
\end{aligned}
$$

where $M_{R}$ and $M_{R_{1}}$ are the corresponding masses in the chiral limit. In the following

we will calculate matrix elements containing resonances within a photon loop. These loops contain divergences that can be absorbed in a counterterm Lagrangian $\mathcal{L}_{\text {c.t. }}$. 
In its general form, this Lagrangian has one term of order $O\left(e^{2}\right)$ and 14 terms of $O\left(e^{2} p^{2}\right)$

$$
\mathcal{L}_{\text {c.t. }}=\hat{C}\left\langle Q U Q U^{\dagger}\right\rangle+\sum_{i=1}^{14} \hat{K}_{i} O_{i} .
$$

The operators $O_{i}$ are identical to those in Eq.(6). The necessary set of Lagrangians for the calculation of processes with resonance-photon loops is given by

$$
\mathcal{L}^{R}=\mathcal{L}_{\text {kin }}^{\gamma}+\mathcal{L}_{\text {kin }}^{R}+\mathcal{L}_{2}^{R}+\mathcal{L}_{2}+\mathcal{L}_{\text {c.t. }} \quad R=V, A, S .
$$

The chiral power counting normally used in the loop expansion of the Goldstone bosons cannot been maintained in the resonance sector. The masses of the resonances do not vanish in the chiral limit, i.e. they have to be considered as a quantity of the order $O(1)$. We therefore expand the loops in the ratio $M_{\pi, K}^{2} / M_{R}^{2}$ and truncate at the order in question. The procedure we use for this purpose is called the heavy mass expansion, see Appendix A for details and examples, where we also discuss the higher order corrections.

The coupling constants in Eq.(9) are not restricted by chiral symmetry, however, they can be related to each other invoking the asymptotic behaviour of QCD and by using large $N_{C}$ arguments [6],

$\left.\begin{array}{ll}\text { Relation } & \text { Source } \\ \hline F_{A}^{2}=F_{V}^{2}-F_{0}^{2} & \text { First Weinberg sum rule [18] } \\ M_{A}^{2} F_{A}^{2}=M_{V}^{2} F_{V}^{2} & \text { Second Weinberg sum rule [18] } \\ F_{V} G_{V}=F_{0}^{2} & \text { Electromagnetic pion form factor } \\ F_{V}=2 G_{V} & \text { Axial pion form factor } \\ M_{S_{1}}=M_{S} \\ \left|\tilde{c}_{d}\right|=\frac{\left|c_{d}\right|}{\sqrt{3}} \\ \left|\tilde{c}_{m}\right|=\frac{\left|c_{m}\right|}{\sqrt{3}}\end{array}\right\} \begin{aligned} & \\ & \end{aligned}$

Note that these relations are valid in the chiral limit only, with the exception of the first Weinberg sum rule that also converges in the presence of quark masses [20]. On the basis of (14) all the parameters in the vector and axial vector sector may be expressed as functions of the pion decay constant $F_{0} \simeq F_{\pi}=92.4 \mathrm{MeV}$ and the rho $\operatorname{mass} M_{V} \simeq M_{\rho}=770 \mathrm{MeV}$,

$$
\begin{array}{lll}
\left|F_{V}\right|=\sqrt{2} F_{0}, & \left|G_{V}\right|=\frac{F_{0}}{\sqrt{2}}, & F_{V} G_{V} 0, \\
\left|F_{A}\right|=F_{0}, & M_{A}=\sqrt{2} M_{V} . &
\end{array}
$$


For a confirmation of the positivity of the product $F_{V} G_{V}$ from experimental data we refer to [21].

In the scalar sector more information is needed to fix $c_{d}$ and $c_{m}$. Following the reasoning in [6] one may turn around the arguments for the scalar contributions in the strong sector (see also the footnote above): the two low energy constants $L_{5}$ and $L_{8}$ are by assumption determined by the resonances, i.e. the finite (experimental) values $L_{5}^{r}(\mu), L_{8}^{r}(\mu)$ fix the couplings $c_{d}, c_{m}$. Therefore at $\mu=M_{\rho}$,

$$
\left|c_{d}\right|=M_{S} \frac{L_{5}^{r}\left(M_{\rho}\right)}{\sqrt{2 L_{8}^{r}\left(M_{\rho}\right)}}, \quad \quad\left|c_{m}\right|=M_{S} \sqrt{2 L_{8}^{r}\left(M_{\rho}\right)}
$$

and with the numerical values (where we omit any errors) $M_{S}=M_{a_{0}}=982 \mathrm{MeV}$, $L_{5}^{r}\left(M_{\rho}\right)=1.4 \times 10^{-3}$ and $L_{8}^{r}\left(M_{\rho}\right)=0.9 \times 10^{-3}$ we arrive at

$$
\left|c_{d}\right|=32 \mathrm{MeV}, \quad \quad\left|c_{m}\right|=42 \mathrm{MeV}, \quad c_{d} c_{m} 0
$$

For a different set of values for $c_{d}, c_{m}$ (due to a different $\left.L_{8}^{r}(\mu)\right)$ see 22]. Note that we will need in our calculation $c_{d}, \tilde{c}_{d}$ only, the couplings of the scalar mesons to the quark masses yields higher order electromagnetic corrections.

For the masses of the pseudoscalar bosons we will use $M_{\pi}=135 \mathrm{MeV}$, $M_{K}=495 \mathrm{MeV}$ and $M_{\eta}=547 \mathrm{MeV}$.

\section{General Procedure}

The low energy coupling constants, introduced in the Chiral Lagrangian $\mathcal{L}_{4}^{Q}$, see (6), are in general divergent. They absorb the divergences at the one-loop level of the generating functional associated with $\mathcal{L}_{2}^{Q}$ [2, 9, 10, 11]. Consequently, they have to be renormalized and the renormalized coupling constants, denoted by $L_{i}^{r}(\mu)$ and $K_{i}^{r}(\mu)$, will depend on the renormalization scale $\mu$.

All low energy parameters of the chiral Lagrangian are determined by the underlying dynamics of QCD and QED. Presently it is not possible to calculate them from first principle. Referring, however, to data from low energy experiments and large $N_{C}$ arguments the values of the $L_{i}^{r}(\mu)$ have been determined in [2]. This is in contrast to the electromagnetic sector, where only an estimate for $K_{8}^{r}(\mu)$ has been given [9], and the determination of the leading order coupling constant $C$ is based on resonance saturation [6].

In order to study the resonant contributions to the $L_{i}^{r}(\mu)$, Ecker et al. [6] separated the low energy coupling constants at a fixed scale point $\mu$ into a sum of resonant $\left(L_{i}^{R}\right)$ and non-resonant $\left(\hat{L}_{i}(\mu)\right)$ contributions,

$$
L_{i}^{r}(\mu)=\sum_{R=V, A, S, P} L_{i}^{R}+\hat{L}_{i}(\mu)
$$


where the sum extends over vector $(\mathrm{V})$, axial vector $(\mathrm{A})$, scalar $(\mathrm{S})$, and pseudoscalar $(\mathrm{P})$ resonances $\mathrm{f}$. Since the resonance exchange occurs at tree level, the corresponding terms $L_{i}^{R}$ are scale independent. The choice of the renormalization scale $\mu$ is not fixed a priori, however, in order to best visualize the contributions from the resonances, $\mu$ should be in the relevant mass region. Indeed, at $\mu=M_{\rho}$ it was found that the finite parts of the strong coupling constants $L_{i}$ are almost determined by resonance exchange, i.e. $\hat{L}_{i}\left(M_{\rho}\right) \simeq 0$ [6] . To be more specific, whenever vector resonances contribute at all, they dominate numerically (for a similar finding see also [17]).

The same ansatz has been used in the electromagnetic sector for the leading order coupling $C$. In contrast to the strong sector resonances contribute to $C$ only within a photon loop, thus the resonant part will be divergent in general. These divergences are absorbed by renormalizing the corresponding non-resonant coupling $\hat{C}$ (which takes the role of a counterterm) and we arrive at the splitting

$$
C=\sum_{R} C^{R}\left(\mu_{0}\right)+\hat{C}^{r}\left(\mu_{0}\right)
$$

where $C^{R}\left(\mu_{0}\right)$ and $\hat{C}^{r}\left(\mu_{0}\right)$ are finite and the scale dependence cancels in the sum.

To be more explicit we take the electromagnetic mass shift of the charged pion at the order $O\left(e^{2}\right)$ as an example. In ordinary CHPT without resonances the charged pion mass reads in the chiral limit (see Eq.(四) above)

$$
\left(M_{\pi^{ \pm}}^{2}\right)_{e . m .}=\frac{2 e^{2}}{F_{0}^{2}} C+O\left(e^{2} m_{q}\right)
$$

entirely determined by the coupling constant $C$. In the resonance approach the pion mass also gets contributions from resonance-photon loops at order $O\left(e^{2}\right)$, see graphs c) and d) in Figure 2 in Appendix A,

$$
\begin{aligned}
\left(M_{\pi^{ \pm}}^{2}\right)_{e . m .}= & -\frac{3 e^{2}}{F_{0}^{2} 16 \pi^{2}}\left[F_{V}^{2} M_{V}^{2}\left(\ln \frac{M_{V}^{2}}{\mu^{2}}+\frac{2}{3}\right)-F_{A}^{2} M_{A}^{2}\left(\ln \frac{M_{A}^{2}}{\mu^{2}}+\frac{2}{3}\right)\right] \\
& +\frac{2 e^{2} \hat{C}}{F_{0}^{2}}-\frac{6 e^{2}}{F_{0}^{2}}\left(F_{V}^{2} M_{V}^{2}-F_{A}^{2} M_{A}^{2}\right) \lambda+O\left(e^{2} m_{q}\right) .
\end{aligned}
$$

The term $\lambda$ is given in Eq.(51) in Appendix A. It contains the loop divergences and produces a pole in $d=4$ dimensions. It can be absorbed by renormalizing the contributions from the non-resonant part,

$$
\hat{C}=\hat{C}^{r}\left(\mu_{0}\right)+3\left(F_{V}^{2} M_{V}^{2}-F_{A}^{2} M_{A}^{2}\right) \lambda .
$$

\footnotetext{
${ }^{\ddagger}$ Recently, the contributions arising from tensor mesons have been determined in CHPT with two light flavours 16 .

${ }^{\S}$ Exceptions to this rule are $L_{5}$ and $L_{8}$, where scalar resonances contribute only. Due to the lack of experimental information in this sector, the resonance saturation hypothesis was assumed, finding good agreement with the decay width $\Gamma\left(a_{0} \rightarrow \pi \eta\right)[6]$.
} 
Invoking Weinberg sum rules [18], see Eq.(14), one circumvents the renormalization of $\hat{C}$, because the loop divergences cancel. The connection to ordinary CHPT without resonances is then given by the relation (19) with the explicit expression for the resonance contributions,

$$
C^{R}=-\frac{3}{2} \frac{F_{V}^{2} M_{V}^{2}}{16 \pi^{2}}\left(\ln \frac{M_{V}^{2}}{M_{A}^{2}}\right),
$$

where again we have used Weinberg sum rules. Putting numbers in (23) and comparing with the observed mass difference $M_{\pi^{ \pm}}^{2}-M_{\pi^{0}}^{2}$ one finds that the resonances nearly saturate the coupling $C$ [6, 7].

For the couplings $K_{i}$ we proceed in a completely analogous way. We split the finite part $K_{i}^{r}(\mu)$ at a fixed scale point $\mu$ in a resonant part and a remainder. The contribution from the resonances $K_{i}^{R}\left(\mu_{0}\right)$ depends on the renormalization scale $\mu_{0}$ at which one subtracts the occurring divergences in the photon-resonance loops,

$$
K_{i}^{r}(\mu)=\sum_{R} K_{i}^{R}\left(\mu_{0}\right)+\hat{K}_{i}^{r}\left(\mu, \mu_{0}\right)
$$

where each term is finite and the $\mu_{0}$ dependence cancels in the sum on the r.h.s. Again, by the use of sum rules we find several combinations of $K_{i}^{R}$ that are finite and thus $\mu_{0}$ independent. But in general, measurable quantities (like the charged pion mass $M_{\pi^{ \pm}}^{2}$ ) involve combinations of $K_{i}^{R}$ that do not show this nice feature and therefore the resonant contributions to these observables remain $\mu_{0}$ dependent [12].

Similarly to the strong sector, we expect the coupling constants $K_{i}^{r}(\mu)$ to be most sensitive to the contributions from the resonances in the proximity of the lightest resonance $\rho$, i.e. we adopt the scale point $\mu=M_{\rho}$ henceforth. Concerning the renormalization scale $\mu_{0}$ in the photon-resonance loops we choose as central value $\mu_{0}=M_{\rho}$ and vary it in the region $M_{\eta} \sim 1 \mathrm{GeV}$ in order to study the scale dependence of the couplings $K_{i}^{R}\left(\mu_{0}\right)$.

In the strong sector and for the leading order electromagnetic coupling $C$ it was found, that there is not much room left for other contributions beside the meson resonances. At $O\left(e^{2} p^{2}\right)$ we have poor informations only (see Section 5) which would establish a similar finding. For the time being, we will treat the finite parts of the renormalized remainder $\hat{K}_{i}^{r}\left(\mu, \mu_{0}\right)$ as unknown parameters.

\section{Resonance Contributions to the $K_{i}^{r}(\mu)$}

In order to determine the resonant contributions to the electromagnetic couplings $K_{i}$, we have calculated $S$-matrix elements with the Lagrangian $\mathcal{L}^{R}$ at the one-loop level and have evaluated the corresponding tree contributions from $\mathcal{L}_{4}^{Q}$. We then split the coupling constants $K_{i}$ according to Eq.(24) in a resonant piece $\left(K_{i}^{R}\right)$ and a remainder $\left(\hat{K}_{i}\right)$. The divergences occurring in the resonant part of the loops are canceled by renormalizing the $\hat{K}_{i}$. The finite part of the resonant loops are finally identified with the corresponding $K_{i}^{R}$ at the scale point $\mu_{0}=M_{\rho}$. 
We have calculated the following set of $S$-matrix elements at the order $O\left(e^{2} p^{2}\right)$ :

- Masses

$$
M_{\pi^{ \pm}}, \quad M_{\pi^{0}}, \quad M_{K^{ \pm}}, M_{K^{0}}, \quad M_{\eta} .
$$

- Scattering Amplitudes (in the chiral limit)

$$
\pi^{+} \pi^{-} \rightarrow \pi^{+} \pi^{-}, \quad \pi^{+} \pi^{-} \rightarrow K^{0} \bar{K}^{0}, \quad \pi^{-} \pi^{0} \rightarrow K^{-} K^{0}
$$

- Matrix Elements (in the chiral limit)

$$
\begin{aligned}
& \left\langle\pi^{-}\left|A_{\mu}^{1-i 2}(0)\right| 0\right\rangle, \quad\left\langle\pi^{+}\left|P^{1+i 2}(0)\right| 0\right\rangle, \quad\left\langle\pi^{-}\left|V_{\mu}^{4-i 5}(0)\right| K^{0}\right\rangle \\
& \left\langle 0\left|T V_{\mu}^{1-i 2}(x) V_{\nu}^{1+i 2}(y)\right| 0\right\rangle, \quad\left\langle 0\left|T A_{\mu}^{1-i 2}(x) A_{\nu}^{1+i 2}(y)\right| 0\right\rangle
\end{aligned}
$$

where

$$
\begin{aligned}
X^{k+i l}(x) & =\bar{q}(x) \Gamma \frac{\lambda_{k}+i \lambda_{l}}{2} q(x), \\
\Gamma & =\left\{\begin{array}{cc}
\gamma_{\mu} & X=V_{\mu} \\
\gamma_{\mu} \gamma_{5} & X=A_{\mu} \\
2 \gamma_{5} & X=P
\end{array}\right.
\end{aligned}
$$

From this list of $S$-matrix elements we have a redundant set of 19 equations for 14 coupling constants. Before presenting the results a few comments are at hand, including some points we have mentioned already before:

1. All one-loop diagrams with resonances have been expanded in powers of $1 / M_{R}^{2}$ with the heavy mass expansion. See Appendix $\mathrm{A}$ for details and examples. In particular, as we have verified, this expansion ensures that the non-local contributions (e.g. to the scattering amplitudes) reduce at order $O\left(e^{2} p^{2}\right)$ to integrals which are independent of the momentum transfer.

2. In order to renormalize the couplings $\hat{K}_{i}$ we work in $d$ dimensions and use the usual modified $\overline{M S}$ scheme, see Eqs.(49-51). The set of matrix elements listed above leads to a over-determined system of equations for the $K_{i}$. We have checked that the renormalization of $\hat{K}_{i}$ is consistent in all processes considered. Since we are only interested in the resonant contributions we will not display them here.

3. The gauge fixing parameter $\lambda$ in the Lagrangian $\mathcal{L}_{\text {kin }}^{\gamma}$ is set to $\lambda=1$ (Feynman gauge). The matching procedure with $S$-matrix elements ensures that we deal with gauge independent quantities. The coupling constants $K_{i}$ themselves may be gauge dependent, since in general they are not observables. 
4. We have checked several parts of our calculation with results given in the literature, namely $(i)$ the resonance-photon loops to $M_{\pi^{ \pm}}^{2}$ and $M_{K^{ \pm}}^{2}$ from 12 restricted to the terms at order $O\left(e^{2} m_{q}\right)$, (ii) the tree level contributions to all the masses from [9, 10], (iii) the tree level calculation for $F_{\pi^{ \pm}}$and the $K_{\ell 3}$ form factor $f_{+}^{K^{0} \pi^{-}}(0)$ at zero momentum transfer from [23].

5. In Figure 11 we give as an example the one-loop contributions to the scattering amplitude $\pi^{-} \pi^{0} \rightarrow K^{-} K^{0}$. Although the Lagrangian $\mathcal{L}_{2}^{R}$ that describes the interaction between Goldstone bosons, photons and resonances is linear in the resonance fields, in some graphs there occur vertices that couple two resonances to Goldstone boson fields. These couplings (with a definite strength) are generated by the covariant derivative (11) acting on the resonances. These diagrams are necessary in order to obtain a consistent determination of the resonance contributions to the $K_{i}$. We have restricted ourselves to the linear resonance model as defined in (9). In particular we have not considered any extension to models with multi-resonance couplings.

6. Since we are only concerned with electromagnetic corrections and work at order $O\left(e^{2} p^{2}\right)$, we have neglected all effects due to the mass difference $m_{u}-m_{d}$, i.e. contributions of the order $O\left[e^{2}\left(m_{u}-m_{d}\right)\right]$ are assumed to be negligible. Throughout we have worked in the isospin limit $m_{u}=m_{d}=\hat{m}$.

7. We would like to emphasize again that the pseudoscalar resonances do not contribute at the order $O\left(e^{2} p^{2}\right)$, thus we conclude that $K_{i}^{P}=0(i=1 \ldots 14)$.

8. In a calculation to the order $O\left(e^{2} m_{q}\right)$ in CHPT with electromagnetic interactions (but without resonances) we may distinguish two kinds of terms: Loop contributions and counterterms (including the $K_{i}$ ). For the matching to the resonant contributions only the terms proportional to the $K_{i}$ are necessary and only those have been calculated. However, one of us has checked that the loop contributions to the electromagnetic mass shifts at order $O\left(e^{2} m_{q}\right)$ are generated by the resonances within photon loops at the two loop level [24].

The full algebraic expressions of the $K_{i}^{R}$ (and for $C$ ) are given in Appendix $\mathrm{B}$. In Table 1 we have listed the values of the $K_{i}^{R}$, which were obtained by using the relations and masses shown in (14) and (15). The coupling $K_{14}^{R}$ has been omitted, since it does not contribute to physical amplitudes.

At first sight we have found that the resonances do not contribute to $K_{7}^{R} \ldots K_{10}^{R}$ at all. Furthermore we find that the vector and axial vector parts, except for $K_{11}^{R}$, are of the same order or even larger as the value $1 /(4 \pi)^{2} \simeq 6.3 \times 10^{-3}$ implied by naive dimensional analysis [25] and dominate the scalar resonant contributions, which are of the order $0.4 \times 10^{-3}$. In most cases the nonvanishing contributions are scale dependent. The scale dependence of $K_{1}^{R} \ldots K_{6}^{R}$ is due to the scalar resonances and that of $K_{11}^{R}$ and $K_{12}^{R}$ is due to the the vector and axial vector contributions. Varying the scale between the values $M_{\eta}$ and $1 \mathrm{GeV}$ causes a change in the central values of 


\begin{tabular}{|l|r|r|r|r|r|}
\hline Type: & \multicolumn{2}{|c|}{$V+A$} & \multicolumn{2}{|c|}{$S+S_{1}$} & Total \\
\hline Units: & $\times 1 /(4 \pi)^{2}$ & $\times 10^{-3}$ & $\times 1 /(4 \pi)^{2}$ & $\times 10^{-3}$ & $\times 10^{-3}$ \\
\hline$K_{1}^{R}\left(\mu_{0}\right)$ & $-\frac{3}{16}(5+\ln 2)$ & -6.8 & $\frac{3}{4} \frac{c_{d}^{2}}{F_{0}^{2}}\left(\ln \frac{M_{S}^{2}}{\mu_{0}^{2}}+\frac{1}{6}\right)$ & 0.4 & -6.4 \\
\hline$K_{2}^{R}\left(\mu_{0}\right)$ & $-\frac{3}{16}(3-\ln 2)$ & -2.7 & $-\frac{3}{4} \frac{c_{d}^{2}}{F_{0}^{2}}\left(\ln \frac{M_{S}^{2}}{\mu_{0}^{2}}+\frac{1}{6}\right)$ & -0.4 & -3.1 \\
\hline$K_{3}^{R}\left(\mu_{0}\right)$ & $\frac{3}{16}(5+\ln 2)$ & 6.8 & $-\frac{3}{4} \frac{c_{d}^{2}}{F_{0}^{2}}\left(\ln \frac{M_{S}^{2}}{\mu_{0}^{2}}+\frac{1}{6}\right)$ & -0.4 & 6.4 \\
\hline$K_{4}^{R}\left(\mu_{0}\right)$ & $-\frac{3}{8}(3-\ln 2)$ & -5.5 & $-\frac{3}{2} \frac{c_{d}^{2}}{F_{0}^{2}}\left(\ln \frac{M_{S}^{2}}{\mu_{0}^{2}}+\frac{1}{6}\right)$ & -0.7 & -6.2 \\
\hline$K_{5}^{R}\left(\mu_{0}\right)$ & $\frac{9}{16}(5+\ln 2)$ & 20.3 & $-\frac{3}{4} \frac{c_{d}^{2}}{F_{0}^{2}}\left(\ln \frac{M_{S}^{2}}{\mu_{0}^{2}}+\frac{1}{6}\right)$ & -0.4 & 19.9 \\
\hline$K_{6}^{R}\left(\mu_{0}\right)$ & $\frac{9}{16}(3-\ln 2)$ & 8.2 & $\frac{3}{4} \frac{c_{d}^{2}}{F_{0}^{2}}\left(\ln \frac{M_{S}^{2}}{\mu_{0}^{2}}+\frac{1}{6}\right)$ & 0.4 & 8.6 \\
\hline$K_{7}^{R} \ldots K_{10}^{R}$ & 0 & 0 & 0 & 0 & 0 \\
\hline$K_{11}^{R}\left(\mu_{0}\right)$ & $\frac{3}{16}\left(\ln \frac{M_{V}^{2}}{\mu_{0}^{2}}+\frac{7}{6}-\ln 2\right)$ & 0.6 & 0 & 0 & 0.6 \\
\hline$K_{12}^{R}\left(\mu_{0}\right)$ & $-\frac{3}{8}\left(\ln \frac{M_{V}^{2}}{\mu_{0}^{2}}+\frac{19}{6}+\ln 2\right)$ & -9.2 & 0 & -9.2 \\
\hline$K_{13}^{R}$ & $\frac{9}{4}$ & 14.2 & 0 & 14.2 \\
\hline
\end{tabular}

Table 1: Contributions from vector and axial vector resonances $(V+A)$, and scalar octet and scalar singlet resonances $\left(S+S_{1}\right)$ to the couplings $K_{1} \ldots K_{13}$. In the second and in the fourth row we list the algebraic expressions, and in the third and in the fifth row we indicate the corresponding numerical values at the scale point $\mu_{0}=M_{V} \simeq M_{\rho}$. In the last row we give the sum of the numerical values. We have used the relations (14) and the numerical values for the parameters given in (15, 17). 
$K_{1}^{R} \ldots K_{6}^{R}$ of roughly $10 \%$. This is due to the smallness of the scalar contributions. The same variation of $\mu_{0}$ causes a change in $K_{12}^{R}$ of about $20 \%$. Only the coupling $K_{11}^{R}$ is very sensitive to the scale point $\mu_{0}$. Moving the scale point between the bounds given above invokes a shift in $K_{11}^{R}$ of the same size as the coupling itself. However, since there is a strong cancellation in $K_{11}^{R}$ between the vector and axial vector resonance contributions, this coupling is small compared to the other coupling constants.

From Table 1 we find that the following linear combinations are scale independent

$$
\begin{aligned}
& S_{1}^{R}=K_{1}^{R}+K_{2}^{R}=-\frac{3}{2} \frac{1}{16 \pi^{2}}, \\
& S_{2}^{R}=K_{5}^{R}+K_{6}^{R}=\frac{9}{2} \frac{1}{16 \pi^{2}}, \\
& S_{3}^{R}=-2 K_{3}^{R}+K_{4}^{R}=-\frac{3}{16 \pi^{2}},
\end{aligned}
$$

where $S_{1} \ldots S_{3}$ have been originally defined in [10]. It is interesting to note that the $\mu_{0}$ independent contributions are pure numbers, an effect of the relations (14) that we have used. The effects of the values we obtained for the $K_{i}^{R}$ are shown in Section 6 .

The behaviour of the $K_{i}$ in the large $N_{C}$ limit [13] is derived in Appendix Q. We have found that the combinations $K_{1}+K_{3}$ and $2 K_{2}-K_{4}$ and the coupling constants $K_{7}$ and $K_{8}$ are suppressed by $1 / N_{C}$ with respect to all the other coupling constants (including $K_{1} \ldots K_{4}$ ). Indeed as one can see from Table 1 the contributions from the resonances vanish in these particular cases. Thus the correct large $N_{C}$ behaviour is a possible hint to resonance saturation in the electromagnetic sector. However, the coupling $K_{8}$ is part of the corrections of order $O\left(e^{2} m_{s}\right)$ to the charged pion mass. In [9] an estimate of the coupling $K_{8}^{r}(\mu)$ was given. There we neglected in the difference $M_{\pi^{ \pm}}^{2}-M_{\pi^{0}}^{2}$ the unknown counterterms proportional to $e^{2} M_{\pi}^{2}$ and fixed the mass difference at its experimental value. We found

$$
K_{8}^{r}\left(M_{\rho}\right)=(-4.0 \pm 1.7) \times 10^{-3}
$$

which is in contrast to our approach, where the coupling $K_{8}$ is not affected by the resonances and thus $K_{8}^{R}=0$. There are two possible answers (that do not exclude each other), namely

(i) the scale point has not been chosen appropriately. Moving to $\mu=M_{\eta}$ we have

$$
K_{8}^{r}\left(M_{\eta}\right)=(-1.4 \pm 1.7) \times 10^{-3}
$$

which is compatible with zero within the error bars.

(ii) There are other contributions that generate the value given in (30), which would show up in the remainder $\hat{K}_{8}$. If we refer to the resonant contributions as 

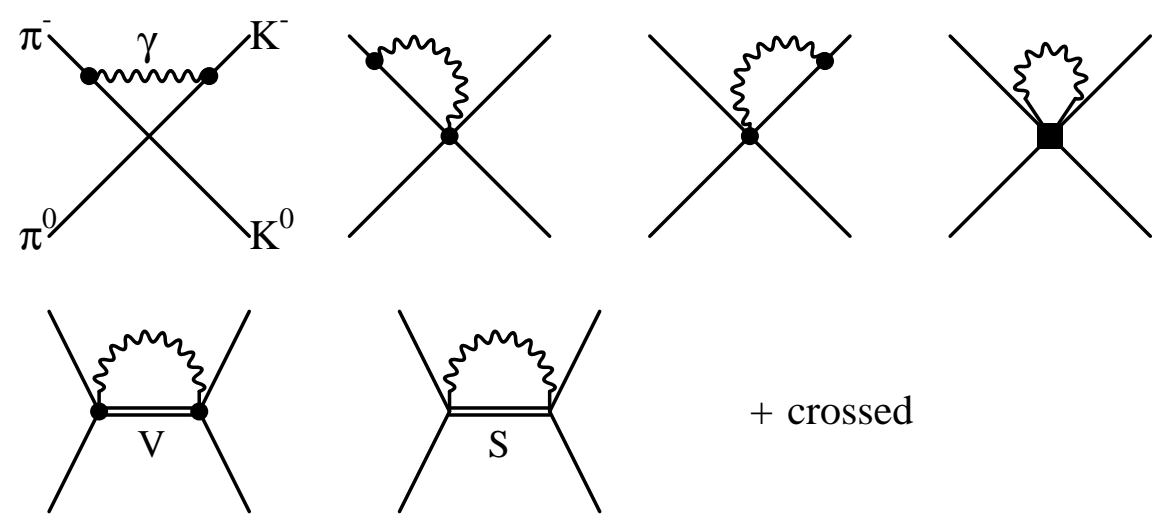

+ crossed
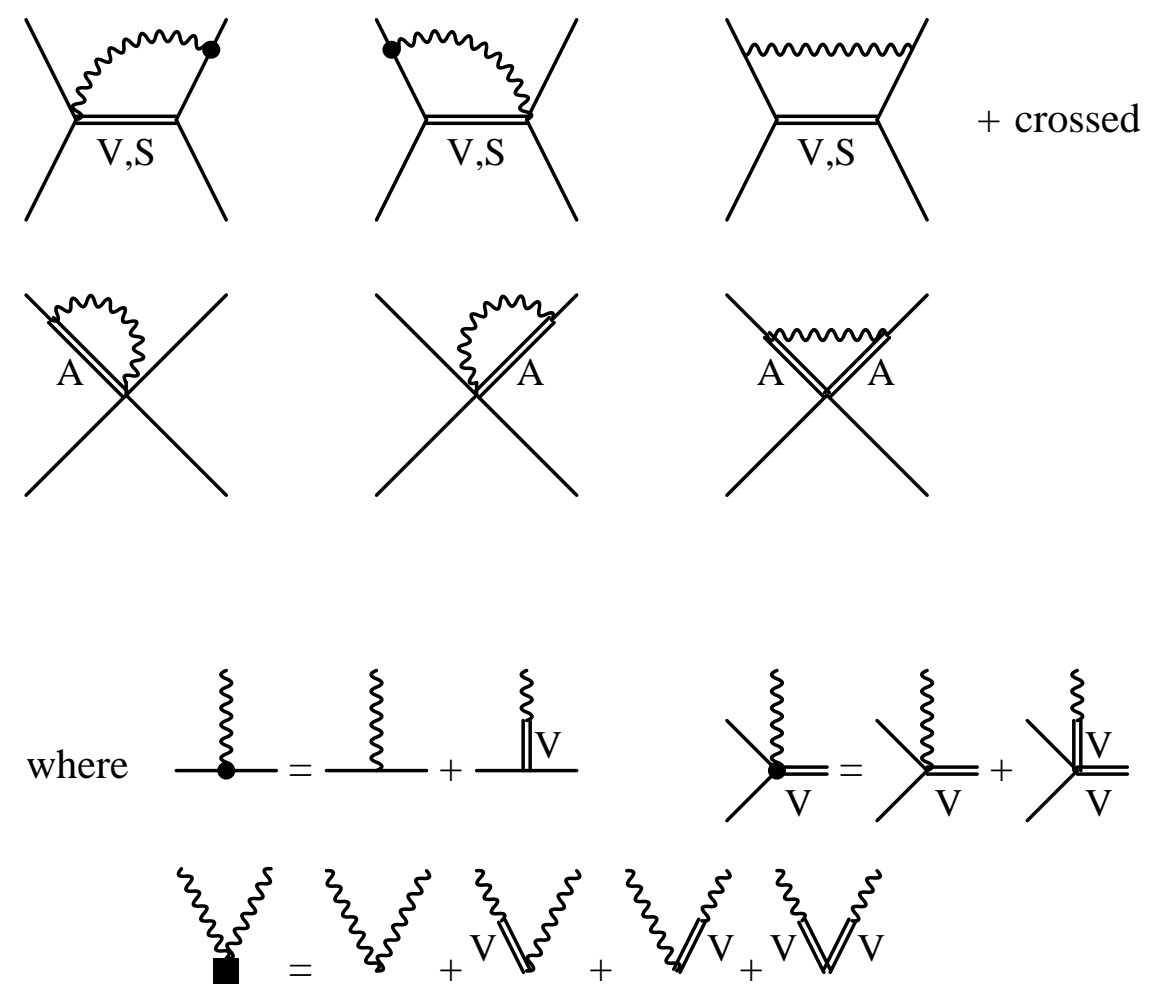

Figure 1: One-loop contributions to the scattering amplitude of $\pi^{-} \pi^{0} \rightarrow K^{-} K^{0}$. The double lines represent the resonances, the letter nearby indicates their type: vector $(V)$, axial vector $(A)$ or scalar $(S)$. Note that the vertices with two resonances are induced by the covariant derivative in (11). 
long distance physics, possible additional contributions could have their origin in the short distance range. Bijnens evaluated the corrections to Dashen's theorem at order $O\left(e^{2} m_{s}\right)$ within the framework of the $1 / N_{C}$ approach [26]. He performed explicitly a short distance calculation of the masses, where no part proportional to $e^{2} m_{s}$ has been found in the contributions to the pion masses. This possibility has therefore no answer yet.

We work at the order $O\left(e^{2} p^{2}\right)$ within the resonance approach determined by the Lagrangians in (9,11). Higher order corrections arise from two sources. (i) The masses and the decay constants of the resonances do not live in a $S U(3)$ invariant world. We know only very little on these corrections, especially for the decay constants. For the contributions to the masses of the vector resonances in the quark mass expansion see [27, [19]. (ii) Higher order terms in the heavy mass expansion may give considerably large corrections to the leading order contribution, in particular if the expansion parameter is $M_{K}^{2} / M_{V}^{2}$, see Example 2 in Appendix A. Thus, when calculating processes with the numbers for the $K_{i}^{R}$ presented above, one should keep in mind the possibility of higher order corrections, especially when kaons are involved.

\section{Applications}

In this section we briefly give the implications of our results on the $K_{i}^{R}$ to several matrix elements and ratios thereof quoted in the literature. We will display the counterterms at order $O\left(e^{2} p^{2}\right)$ proportional to $K_{i}$ only, for the full expressions we refer to the indicated literature.

There are three combinations of form factors and decay constants that involve the same counterterms [10, 23],

$$
\begin{aligned}
\left.r_{K \pi}\right|_{\text {Res. }} & =\left.\frac{f_{+}^{K^{+} \pi^{0}}(0)}{f_{+}^{K^{0} \pi^{-}}(0)}\right|_{\text {Res. }}=\frac{4}{9} e^{2} \frac{M_{K}^{2}}{M_{\eta}^{2}-M_{\pi}^{2}}\left(2 S_{2}^{R}+3 S_{3}^{R}\right) \\
\left.f_{+}^{\eta \pi^{+}}(0)\right|_{\text {Res. }} & =\left.\frac{1}{\sqrt{3}} r_{K \pi}\right|_{\text {Res. }} \\
\left.\frac{F_{K^{0}} F_{\pi^{ \pm}}}{F_{K^{ \pm}} F_{\pi^{0}}\left(\lambda_{3} / \sqrt{2}\right)}\right|_{\text {Res. }} & =-\frac{1}{3} e^{2}\left(2 S_{2}^{R}+3 S_{3}^{R}\right),
\end{aligned}
$$

where $f_{+}^{P \pi}(0)$ is the form factor associated with the momentum $\left(p_{P}+p_{\pi}\right)_{\mu}$ in the decay $P \rightarrow \pi \ell \bar{\nu}_{\ell}$ at zero momentum transfer and $F_{P}$ is the $P_{\ell 2}$ decay constant. From Eq.(29) we find immediately that $2 S_{2}^{R}+3 S_{3}^{R}=0$, therefore the above quantities do not receive contributions from the resonances. Next we consider the resonant part of the electromagnetic corrections to the amplitude $\eta \rightarrow 3 \pi$ [11],

$$
\begin{aligned}
\left.A_{Q E D}(s, t, u)\right|_{\text {Res. }}= & -\frac{2}{9 \sqrt{3}} \frac{e^{2}}{F_{0}^{2}} M_{\pi}^{2}\left[1+\frac{3\left(s-M_{\pi}^{2}\right)-M_{\eta}^{2}}{M_{\eta}^{2}-M_{\pi}^{2}}\right] \\
& \times\left(2 S_{2}^{R}+3 S_{3}^{R}-2 K_{9}^{R}-2 K_{10}^{R}\right),
\end{aligned}
$$


which again vanish in the resonance approach.

Finally, we discuss the resonant contributions to the masses. We start with the masses of the neutral particles [9],

$$
\begin{aligned}
\left.M_{\pi^{0}}^{2}\right|_{\text {Res. }}= & -\frac{2}{9} e^{2} M_{\pi}^{2}\left(12 S_{1}^{R}+10 S_{2}^{R}+9 S_{3}^{R}-12 K_{7}^{R}-12 K_{8}^{R}-10 K_{9}^{R}-10 K_{10}^{R}\right) \\
\left.M_{K^{0}}^{2}\right|_{\text {Res. }}= & -\frac{8}{9} e^{2} M_{K}^{2}\left(3 S_{1}^{R}+S_{2}^{R}-3 K_{7}^{R}-3 K_{8}^{R}-K_{9}^{R}-K_{10}^{R}\right) \\
\left.M_{\eta}^{2}\right|_{\text {Res. }}= & \frac{2}{9} e^{2}\left[2 M_{\pi}^{2}\left(K_{9}^{R}+K_{10}^{R}\right)-M_{\eta}^{2}\left(12 S_{1}^{R}+6 S_{2}^{R}+3 S_{3}^{R}-12 K_{7}^{R}\right.\right. \\
& \left.\left.\quad-12 K_{8}^{R}-4 K_{9}^{R}-4 K_{10}^{R}\right)\right] .
\end{aligned}
$$

As the quantities in the previous examples, the masses of the neutral particles are unaffected by the resonances, whereas the masses of the charged particles have the form,

$$
\begin{aligned}
& \left.M_{\pi^{ \pm}}^{2}\right|_{R e s .}\left(\mu_{0}\right)=4 e^{2}\left\{2 M_{K}^{2} K_{8}^{R}-\frac{1}{9} M_{\pi}^{2}\left[6 S_{1}^{R}+5 S_{2}^{R}-6 K_{7}^{R}-15 K_{8}^{R}\right.\right. \\
& \left.\left.-5 K_{9}^{R}-23 K_{10}^{R}-18 K_{11}^{R}\left(\mu_{0}\right)\right]\right\} \\
& \left.M_{K^{ \pm}}^{2}\right|_{\text {Res. }}\left(\mu_{0}\right)=\frac{4}{3} e^{2}\left\{M_{\pi}^{2}\left(3 K_{8}^{R}+K_{9}^{R}+K_{10}^{R}\right)\right. \\
& -\frac{1}{3} M_{K}^{2}\left[6 S_{1}^{R}+5 S_{2}^{R}-6 K_{7}^{R}-24 K_{8}^{R}\right. \\
& \left.\left.-2 K_{9}^{R}-20 K_{10}^{R}-18 K_{11}^{R}\left(\mu_{0}\right)\right]\right\} \text {. }
\end{aligned}
$$

They receive contributions from the resonances and all the scale dependence is concentrated in $K_{11}^{R}\left(\mu_{0}\right)$. Putting in numbers, we observe that the corrections to $M_{\pi^{ \pm}}^{2}$ and $M_{K^{ \pm}}^{2}$ are proportional to $M_{\pi}^{2}$ and $M_{K}^{2}$, respectively, and that they contain both the same coefficient,

$$
\begin{aligned}
\left.\frac{M_{\pi^{ \pm}}^{2}}{M_{\pi}^{2}}\right|_{\text {Res. }}\left(\mu_{0}\right) & =\left.\frac{M_{K^{ \pm}}^{2}}{M_{K}^{2}}\right|_{\text {Res. }}\left(\mu_{0}\right) \\
& =-\frac{1}{64 \pi^{2}} e^{2}\left[17+6\left(\ln 2-\ln \frac{M_{V}^{2}}{\mu_{0}^{2}}\right)\right] .
\end{aligned}
$$

Now we are able to discuss the different terms that contribute to the corrections to Dashen's theorem [14, 15], that involves the electromagnetic mass differences $\left(M_{K^{ \pm}}^{2}-M_{K^{0}}^{2}\right)_{e . m .}-\left(M_{\pi^{ \pm}}^{2}-M_{\pi^{0}}^{2}\right)_{e . m .}=\left(\Delta M_{K}^{2}-\Delta M_{\pi}^{2}\right)_{e . m .}$ at order $O\left(e^{2} m_{q}\right)$. We do not show the algebraic expression here, in its full form it may be found in [9]. We distinguish two different parts, one is generated by the photon loop and the counterterms $K_{i}$, the other part is due to mesonic loops and to the counterterm $L_{5}$ and therefore proportional to the coupling $C$. The two parts are then again separated in contributions from the loops and from the counterterms, respectively. As an ingredient we need the value of $L_{5}^{r}(\mu)$ [2],

$$
L_{5}^{r}(\mu)=(1.4 \pm 0.5) \times 10^{-3}-\frac{3}{128 \pi^{2}} \ln \frac{\mu}{M_{\rho}} .
$$




\begin{tabular}{|c|r|r|r|r|r|}
\hline \multicolumn{5}{|c|}{$\left(\Delta M_{K}^{2}-\Delta M_{\pi}^{2}\right)_{e . m .} \quad\left[\times 10^{-3}(\mathrm{GeV})^{2}\right]$} \\
\hline & \multicolumn{2}{|c|}{$\sim e^{2} m_{q}$} & \multicolumn{2}{|c|}{$\sim e^{2} \frac{C}{F_{0}^{4}} m_{q}$} & \multirow{2}{*}{} \\
\cline { 2 - 5 }$\mu$ & Loops & $\Delta R_{\text {Res. }}$ & Loops & $L_{5}^{r}$ & Total \\
\hline$M_{\eta}$ & 0.52 & -0.70 & -0.04 & -0.67 & -0.88 \\
\hline$M_{\rho}$ & 0.79 & -0.70 & 0.11 & -0.43 & -0.21 \\
\hline $1 \mathrm{GeV}$ & 1.0 & -0.70 & 0.23 & -0.24 & 0.30 \\
\hline Errors & & ${ }_{-0.10}^{+0.13}$ & & \pm 0.15 & ${ }_{-0.25}^{+0.28}$ \\
\hline
\end{tabular}

Table 2: Corrections to Dashen's theorem at order $O\left(e^{2} m_{q}\right)$ at different scale points $\mu$. The contributions from the resonances $\Delta R_{\text {Res. }}$ are $\mu$ - independent and renormalized at $\mu_{0}=M_{\rho}$. The error of this quantity reflects the variation of the renormalization scale $\mu_{0}$ from $M_{\eta}(+)$ up to $1 \mathrm{GeV}(-)$. The error of $L_{5}^{r}$ is $\mu$ independent.

The numerical results are presented in Table 2 for the three scale points $\mu=$ $\left(M_{\eta}, M_{\rho}, 1 \mathrm{GeV}\right)$. The contributions from the resonances, shown as counterterms $\Delta R_{\text {Res. }}$, depend on the renormalization scale $\mu_{0}$ that we choose to be $\mu_{0}=M_{\rho}$. The variation of this scale is reflected in the error of this value, namely the upper bound corresponds to $\mu_{0}=M_{\eta}$, the lower bound to $\mu_{0}=1 \mathrm{GeV}$, respectively. We obtain

$$
\Delta R_{\text {Res. }}=\left(-0.70_{-0.10}^{+0.13}\right) \times 10^{-3}(\mathrm{GeV})^{2} .
$$

From the results in Table 2 we see that all the possible contributions are of the same magnitude, none of them is negligible. Considering a scale point in the range $0.7 \sim 1 \mathrm{GeV}$, the uncertainty that enters the calculation at the order $O\left(e^{2} m_{q}\right)$ is of the same size as the central value itself.

In one place we are able to check the size of higher order corrections. In 12 (see also [28]) we have calculated with the Lagrangians in (9,11) the contributions from the resonances to electromagnetic mass differences without truncating the integrals at the order $O\left(e^{2} m_{q}\right)$. At the scale point $\mu=\mu_{0}=M_{\rho}$ we have found for the corrections to Dashen's theorem (transformed to the present values for the parameters)

$$
\left(\Delta M_{K}^{2}-\Delta M_{\pi}^{2}\right)_{e . m .}\left(M_{\rho}\right)=0.37 \times 10^{-3}(\mathrm{GeV})^{2} \quad \text { in [12]. }
$$

Note that this number is very sensitive to the choice of the parameters in the resonance sector. In Table 2 the corresponding result is the sum of the contributions to 
the photon loop and $\Delta R_{\text {Res. }}$, namely

$$
\left.\left(\Delta M_{K}^{2}-\Delta M_{\pi}^{2}\right)\right|_{\gamma-\text { loop }+ \text { Res. }}\left(M_{\rho}\right)=0.09 \times 10^{-3}(\mathrm{GeV})^{2}
$$

at the same scale and renormalization point as above. Thus the higher order terms from the exact evaluation of the integral gives $40 \%$ corrections to $\Delta R_{R e s}$ as it could be expected from Example 2 in Appendix A. Due to the strong cancellation in the sum of the loop and counterterm contributions, it completely reveals the number of this part and moves as a consequence the central value of the final result to the positive region. Taking this higher order corrections explicitly into account we arrive at

$$
\begin{aligned}
& \left(\Delta M_{K}^{2}-\Delta M_{\pi}^{2}\right)_{e . m .}\left(M_{\rho}\right)= \\
& \quad\left(0.07_{-0.25}^{+0.28}\right) \times 10^{-3}(\mathrm{GeV})^{2}+\Delta R_{\text {Non-res. }}\left(M_{\rho}\right)+O\left(e^{2} m_{q}^{2}\right)
\end{aligned}
$$

where $\Delta R_{N o n-r e s .}$ are the non-resonant contributions at the order $O\left(e^{2} m_{q}\right)$ determined by the $\hat{K}_{i}\left(\mu, \mu_{0}\right)$ defined in (24) that we do not consider here. Their determination is beyond the scope of this work.

In summary we find that all the contributions to the corrections to Dashen's theorem that we take into account are of the same size. Furthermore we find that there occur strong cancellations in summing up all the parts, leading to the result that the uncertainties at the order $O\left(e^{2} m_{q}\right)$ and higher order corrections could easily reveal the central values quoted in Table 2; the same may be valid for non-resonant contributions $\Delta R_{N o n-r e s .}$. Thus from our calculation we may conclude that the low energy contributions to the violation of Dashen's theorem are small.

\section{Conclusions}

1. Within the framework of chiral perturbation theory we have considered the coupling constants $K_{i}(i=1 \ldots 14)$ in the electromagnetic interaction Lagrangian $\mathcal{L}_{4}^{Q}$ at order $O\left(e^{2} p^{2}\right)$ [9, 10, 11]. We have determined the contributions to the $K_{i}$ that arise from resonances within a photon loop at order $O\left(e^{2} p^{2}\right)$ by calculating masses, scattering amplitudes and matrix elements with external currents (see Section 5). For the interactions of the pseudoscalar mesons with the resonances we have used the lowest order interaction Lagrangian that is linear in the resonance fields, introduced by Ecker et al. [6]. Throughout we have worked in the isospin limit $m_{u}=m_{d}=\hat{m}$ (i.e. neglecting contributions of the order $\left.O\left[e^{2}\left(m_{u}-m_{d}\right)\right]\right)$.

2. Formally, we have split the contributions to the $K_{i}$ in a resonant part $K_{i}^{R}$, which we have calculated, and a remainder $\hat{K}_{i}$. In general the resonancephoton loops generate ultraviolet divergences that are absorbed by renormalization of the corresponding $\hat{K}_{i}$ at a specific scale point $\mu_{0}$. There are no contributions to $K_{7} \ldots K_{10}$ from the resonances at all (see Table 11). For the remaining $K_{i}^{R}$ we have 
found that the vector and axial vector parts, except for $K_{11}^{R}$, are of the same order or even larger as the value $1 /(4 \pi)^{2} \simeq 6.3 \times 10^{-3}$ implied by naive dimensional analysis [25]. Whenever the scalars contribute, they generate small but scale dependent shifts. Since the scalar contributions are small, the values of $K_{1}^{R} \ldots K_{6}^{R}$ are hardly affected by the scale dependence. This is in contrast to the case of $K_{11}^{R}$. Here the scale dependence is due to the vector and axial vector contributions. The constant $K_{11}^{R}$ is very sensitive to the choice of the scale point $\mu_{0}$. Varying $\mu_{0}$ between the values $M_{\eta}$ and $1 \mathrm{GeV}$ gives rise to a shift in $K_{11}^{R}$ of the same size as the coupling itself. However, since there is a strong cancellation in $K_{11}^{R}$ between the vector and axial vector resonance contributions, this coupling is small compared to the other coupling constants. The scale dependence in the coupling $K_{12}^{R}$ is also generated by the vector and axial vector contributions and the variation of $\mu_{0}$ between the bounds given above causes a shift of about $20 \%$.

3. Comparing our results to the expectation from the large $N_{C}$ limit (see Appendix (C) we find agreement for the four linear combinations of couplings that are suppressed in this limit. The couplings $K_{7}$ and $K_{8}$ do not get any contributions from the resonances at all. The combinations $K_{1}^{R}+K_{3}^{R}$ and $2 K_{2}^{R}-K_{4}^{R}$ vanish, even though the individual couplings $K_{1} \ldots K_{4}$ each get large contributions from the resonances.

4. In the evaluation of the resonance contributions to the $K_{i}$ we have used the heavy mass expansion (see Appendix A) in order to truncate the integrals at the order $O\left(e^{2} p^{2}\right)$. We have found that higher order corrections can be sizeable, especially when the ratio $M_{K}^{2} / M_{V}^{2}$ is involved as expansion parameter, see Example 2 in Appendix A. Thus when calculating processes with the values for the $K_{i}^{R}$ presented in this article, one should keep in mind the possibility of higher order corrections, in particular when kaons are involved.

5. As an application we have discussed the effects of our result on the corrections to Dashen's theorem [14, 15] at the order $O\left(e^{2} m_{q}\right)$. We find a strong cancellation in the sum of the different terms leading to the result that the uncertainties are of the same order of magnitude than the central value at the scale point $\mu=M_{\rho}$ (the same holds for all the scale points in the range $0.7 \sim 1 \mathrm{GeV}$ ),

$$
\begin{aligned}
& \left(\Delta M_{K}^{2}-\Delta M_{\pi}^{2}\right)_{e . m .}\left(M_{\rho}\right)= \\
& \quad\left(0.07_{-0.25}^{+0.28}\right) \times 10^{-3}(\mathrm{GeV})^{2}+\Delta R_{\text {Non-res. }}\left(M_{\rho}\right)+O\left(e^{2} m_{q}^{2}\right)
\end{aligned}
$$

where $\Delta R_{N o n-r e s .}$ are the non-resonant contributions at the order $O\left(e^{2} m_{q}\right)$ determined by the $\hat{K}_{i}$. Their inclusion is beyond the scope of this work. The error indicated is due to the strong coupling constant $L_{5}^{r}\left(M_{\rho}\right)$ and the variation of the renormalization scale $\mu_{0}$ for the resonance contributions in the range $M_{\eta} \sim 1 \mathrm{GeV}$. Note that in the numerical part in (42) the higher order corrections due to the heavy mass expansion are already included [12]. 
After completing this article we became aware of a work by Bijnens and Prades [35 on the same subject. The authors have considered the electromagnetic interaction Lagrangian $\mathcal{L}_{4}^{Q}$ including the $\eta^{\prime}$ as an additional degree of freedom. Within the $1 / N_{C}$ approach [8, 26, 36] they have calculated the short distance contributions to all the coupling constants $K_{i}$ at leading order in $1 / N_{C}$ and the long distance part of the linear combinations of $K_{i}$ needed for the electromagnetic mass corrections. They found a large violation of Dashen's theorem,

$$
\left(\Delta M_{K}^{2}-\Delta M_{\pi}^{2}\right)_{e . m .}=(1.06 \pm 0.32) \times 10^{-3}(\mathrm{GeV})^{2} .
$$

The source of the large difference to our result may be identified easily. The contributions from the $K_{i}$ (that we call $\Delta R$ ) is positive in their approach [35,

$$
\Delta R=0.53 \times 10^{-3}(\mathrm{GeV})^{2},
$$

whereas the corresponding value of our calculation is given in Eq.(38), $\Delta R_{\text {Res. }}=$ $-0.70 \times 10^{-3}(\mathrm{GeV})^{2}$. Since their technique involves an Euclidean cut-off, the comparison to our procedure cannot be carried out directly. It will need some more work to find the connection between the two approaches.

Even more recently two other preprints by Gao, Yan and Li [37] and by Donoghue and Pérez [38] concerning the electromagnetic mass differences came to our attention. In [37] the authors consider an $U(3)_{R} \times U(3)_{L}$ effective field theory containing the low-lying pseudoscalar, vector and axial vector mesons. The divergences that occur in the resonance-photon loop contributions to the electromagnetic mass corrections are absorbed by using an intrinsic parameter $g$ of the theory [37. The corrections to Dashen's theorem are found to be large,

$$
\left(\Delta M_{K}^{2}-\Delta M_{\pi}^{2}\right)_{e . m .}=1.08 \times 10^{-3}(\mathrm{GeV})^{2} .
$$

In [38] the Cottingham method [39] is used in order to calculate the electromagnetic mass corrections. This approach leads to a dispersion integral over the Compton scattering amplitudes $\gamma(\pi, K) \rightarrow \gamma(\pi, K)$. As input serves CHPT for the elastic scattering, inelastic production of the low-lying resonances and experimental data in the intermediate energy region, and perturbative QCD at high energies. The authors have found substantial contributions to the electromagnetic kaon mass difference mainly due to the effect of the kaon mass in the propagator of the Born term in the $\gamma K$ scattering amplitude [38] (this corresponds to the contribution from graph a) in Figure 2 in the present work). Correspondingly the corrections to Dashen's theorem are also large,

$$
\left(\Delta M_{K}^{2}-\Delta M_{\pi}^{2}\right)_{e . m .}=(1.34 \pm 0.61) \times 10^{-3}(\mathrm{GeV})^{2},
$$

where we combined the individual electromagnetic mass differences and added the errors in quadrature. One difference to our work may be traced out in the resonance region, where in [38] the experimental masses and decay constants have been applied (including finite widths), whereas we work in the $S U(3)$ limit and neglect the widths of the resonances. Whether this different treatment of the resonances is essential remains to be studied. 


\section{Acknowledgments}

We are grateful to J. Bijnens, J. Gasser and D. Wyler for very helpful discussions. R.U. would like to thank Matthias Steinhauser for his never ending patience to explain the virtue of the heavy mass expansion.

\section{A Heavy Mass Expansion}

With the heavy mass expansion loop integrals may be conveniently simplified, in the case when all the internal masses can be divided into a set of large $\underline{M}=\left\{M_{1}, M_{2}, \ldots\right\}$ and small $\underline{m}=\left\{m_{1}, m_{2}, \ldots\right\}$, and when all external momenta $\underline{q}=\left\{q_{1}, q_{2}, \ldots\right\}$ are small compared to the scale of the large masses $\underline{M}$. The heavy mass expansion was first described in an equivalent form in [29] and was mathematically rigorously formulated in [30, 31, 32]. Let $F_{\Gamma}$ be the Feynman integral associated with the Feynman diagram $\Gamma$, then the proposition is valid 33.

$$
F_{\Gamma} \stackrel{M}{\rightarrow}=\infty \sum_{\gamma} F_{\Gamma / \gamma} \circ T_{\underline{q}^{\gamma}, \underline{m}^{\gamma}} F_{\gamma}\left(\underline{q}^{\gamma}, \underline{m}^{\gamma}, \underline{M}\right)
$$

where the sum is over all subgraphs $\gamma$ of $\Gamma$ such that each $\gamma$

a) contains all lines with heavy masses,

b) consists of connectivity components that are one-particle-irreducible with respect to lines with small masses.

The operator $T$ performs Taylor expansion in the variables $\underline{q}^{\gamma}$ and $\underline{m}^{\gamma}$. It is understood that the operator $T$ acts on the integrand of the subgraph $\gamma$ thus producing a polynomial $P_{\gamma}$ in $\underline{q}^{\gamma}$ and $\underline{m}^{\gamma}$. The momenta $\underline{q}^{\gamma}$ denotes the external momenta of the subgraph $\gamma$. It should be noted that the external momenta $\underline{q}^{\gamma}$ is defined with respect to $\gamma$ and thus in general consists of some genuine external momenta, shared by the whole Feynman diagram $\Gamma$ and $\gamma$, as well as momenta flowing through internal lines of $\Gamma$, which are external ones of $\gamma$. The masses $\underline{m}^{\gamma}$ collects all light masses in the subgraph $\gamma$. The notation $F_{\Gamma / \gamma}$ means that the subgraph $\gamma$ is contracted to a single vertex $v_{\gamma}$. After insertion of the polynomial $P_{\gamma}$ in $v_{\gamma}$ the new Feynman integral $F_{\Gamma / \gamma} \circ P_{\gamma}$ must be evaluated. All possible subgraphs $\gamma$ must be found and their contribution must be added to the result. Writing the Taylor expansion operator as

$$
T_{x_{1}, x_{2}, \ldots}=\sum_{n=0}^{\infty} t^{n}\left(x_{1}, x_{2}, \ldots\right)
$$

then one immediately sees that

$$
t^{n}\left(\underline{q}^{\gamma}, \underline{m}^{\gamma}\right) F_{\gamma}\left(\underline{q}^{\gamma}, \underline{m}^{\gamma}, \underline{M}\right)
$$

scales like $M^{d(\gamma)-n}$, where $d(\gamma)$ is the mass dimension of the subgraph $\gamma$. 


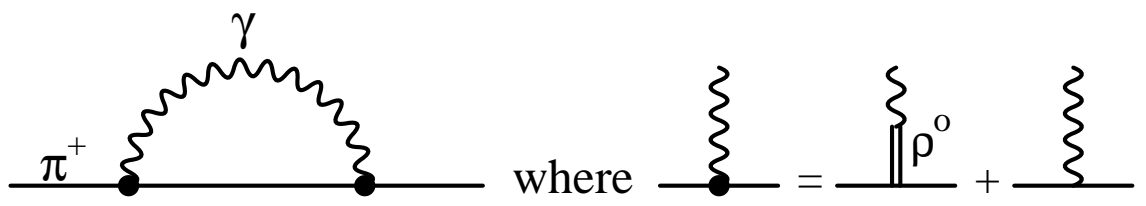

a)

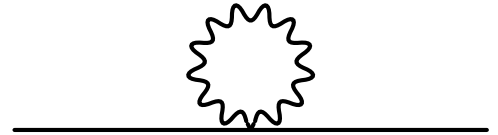

b)

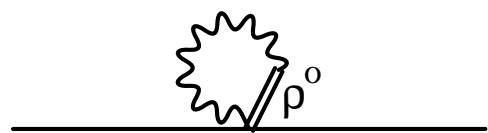

c)

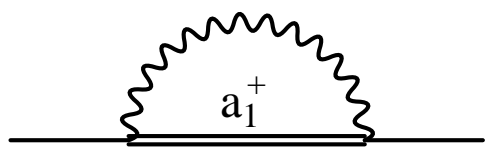

d)

Figure 2: One-loop contributions to the electromagnetic mass shift of $\pi^{ \pm}$.

Example 1: Consider the loop contribution to $M_{\pi^{ \pm}}^{2}$ containing the axial vector resonance $a_{1}$ (with mass $M_{A}$ ) shown in Figure 2 $\mathrm{d}$ ). This diagram can be worked out exactly and gives [12]

$$
\begin{aligned}
F_{\Gamma}= & (3-\epsilon) \frac{e^{2} F_{A}^{2}}{F_{0}^{2}} \frac{1}{i} \int \frac{d^{4} q}{(2 \pi)^{4}} \frac{1}{M_{A}^{2}-q^{2}} \\
& +\frac{e^{2} F_{A}^{2}}{F_{0}^{2}} \frac{1}{i} \int \frac{d^{4} q}{(2 \pi)^{4}} \frac{q^{2}\left[M_{\pi}^{2}+(3-\epsilon) \nu\right]+(2-\epsilon) \nu^{2}}{q^{2}\left[M_{A}^{2}-(q+p)^{2}\right]},
\end{aligned}
$$

where $\epsilon=4-d$ (d: dimension), $\nu=p q$ and $p$ is the pion momentum. The integrals are easily evaluated,

$$
\begin{aligned}
F_{\Gamma}= & \frac{3 e^{2}}{F_{0}^{2} 16 \pi^{2}}\left[F_{A}^{2} M_{A}^{2}\left(\ln \frac{M_{A}^{2}}{\mu_{0}^{2}}+\frac{2}{3}\right)\right]+\frac{6 e^{2}}{F_{0}^{2}} F_{A}^{2} M_{A}^{2} \lambda \\
& -\frac{e^{2} F_{A}^{2}}{F_{0}^{2} 16 \pi^{2}} M_{\pi}^{2}\left[2+\frac{3}{2} \ln \frac{M_{A}^{2}}{\mu_{0}^{2}}+I_{1}\left(\frac{M_{\pi}^{2}}{M_{A}^{2}}\right)\right],
\end{aligned}
$$




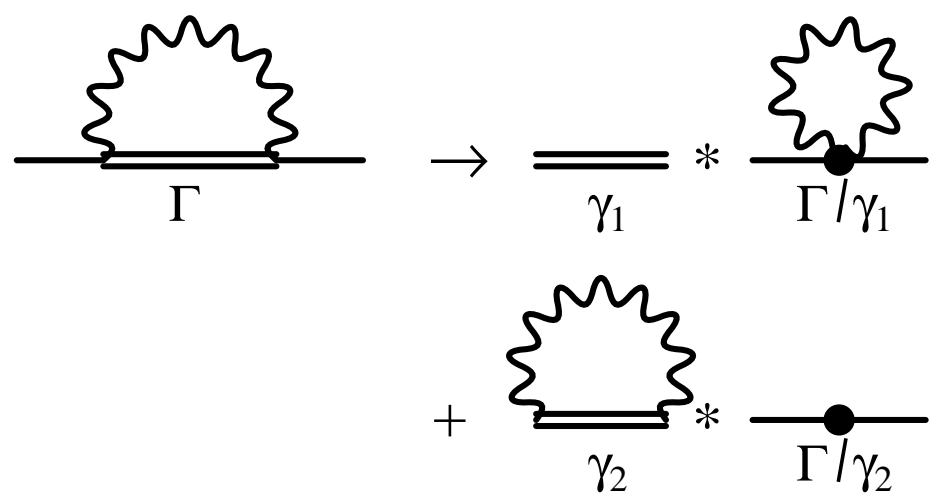

Figure 3: Example of the heavy mass expansion.

with

$$
\begin{aligned}
\lambda & =\frac{\mu_{0}^{d-4}}{16 \pi^{2}}\left\{\frac{1}{d-4}-\frac{1}{2}\left[\ln 4 \pi+\Gamma^{\prime}(1)+1\right]\right\} \\
I_{1}(z) & =\int_{0}^{1} x \ln [x-x(1-x) z] d x .
\end{aligned}
$$

In the heavy mass expansion two subgraphs $\gamma_{1}$ and $\gamma_{2}$ are identified, see Figure 3 . The first subdiagram $\gamma_{1}$ is found to be the heavy mass propagator. It is characterized by $q \ll M_{A}$. The external momentum flowing through the heavy propagator is the loop momentum $q$. We thus expand $\gamma_{1}$ in powers of $q$. Working up to order $O\left(1 / M_{A}^{2}\right)$ we get

$$
P_{\gamma_{1}}=T_{k} F_{\gamma_{1}}=\frac{1}{M_{A}^{2}}+O\left(\frac{q^{2}}{M_{A}^{2}}\right) .
$$

This term corresponds to the naive expansion of a Feynman integral in powers of $1 / M_{A}^{2}$. The diagram $\Gamma / \gamma_{1}$ vanishes, however, in dimensional regularization, because only the massless photon runs through the loop. The second subgraph $\gamma_{2}$ is the loop itself. $\gamma_{2}$ is characterized by $p \ll q, M_{A}$ and if the photon had a mass $m_{\gamma}$ by $m_{\gamma}, p \ll q, M_{A}$. Expanding in $\gamma_{2}$ the heavy propagator in $p$ to the order $O\left(1 / M_{A}^{2}\right)$ one obtains

$$
\begin{aligned}
\sum_{\gamma} F_{\Gamma / \gamma} \circ T_{\underline{q}^{\gamma}, \underline{m}^{\gamma}}= & F_{\Gamma / \gamma_{2}} T_{p} F_{\gamma_{2}} \\
= & \frac{3 e^{2}}{F_{0}^{2} 16 \pi^{2}}\left[F_{A}^{2} M_{A}^{2}\left(\ln \frac{M_{A}^{2}}{\mu_{0}^{2}}+\frac{2}{3}\right)\right]+\frac{6 e^{2}}{F_{0}^{2}} F_{A}^{2} M_{A}^{2} \lambda \\
& -\frac{e^{2} F_{A}^{2}}{F_{0}^{2} 16 \pi^{2}} M_{\pi}^{2}\left[2+\frac{3}{2} \ln \frac{M_{A}^{2}}{\mu_{0}^{2}}-\frac{1}{4}\right]
\end{aligned}
$$


As can be seen from the equations (50) and (53) one has to compare the numerical value of $I_{1}\left(M_{\pi}^{2} / M_{A}^{2}\right)$ with $-1 / 4$. We may split the integral $I_{1}$ in a part at $z=0$ and a remainder $\bar{I}_{1}(z)=I_{1}(z)-I_{1}(0)$. Numerically we find

$$
\begin{aligned}
I_{1}\left(\frac{M_{\pi}^{2}}{M_{A}^{2}}\right) & =I_{1}(0)+\bar{I}_{1}\left(\frac{M_{\pi}^{2}}{M_{A}^{2}}\right) \\
& =-0.250-0.003
\end{aligned}
$$

where we have used the relations in (15). Thus in our case the result from the heavy mass expansion in (53) corresponds to $I_{1}(0)$. The difference $\bar{I}_{1}(z)$ to the exact result is small. Indeed, inserting in (50) one gets for the expression in the parentheses on the last line,

$$
\left.\left[2+\frac{3}{2} \ln \frac{M_{A}^{2}}{\mu_{0}^{2}}+I_{1}(0)+\bar{I}_{1}\left(\frac{M_{\pi}^{2}}{M_{A}^{2}}\right)\right]\right|_{\mu_{0}=M_{\rho}}=2.790-0.003,
$$

where we have split the remainder from all the other contributions and fixed the renormalization scale point at $\mu_{0}=M_{\rho}$.

Example 2: In the previous example we have considered an integral that converges well in the $1 / M_{R}$ expansion, i.e. the truncation after the leading term gives a good description of the entire integral. In general, we cannot expect that this approximation of a loop contribution works so well. In the present example we give an estimate of the upper bound for the error that we induce with the truncation. We consider again the loop contributions in Figure 2, but this time we choose the part a) and calculate its contribution to $M_{K^{ \pm}}^{2}$. The expansion parameter is therefore $M_{K}^{2} / M_{V}^{2} \sim 0.4$, and the relevant expression reads [12],

$$
\begin{aligned}
F_{\Gamma} & =\frac{1}{8 \pi^{2}} e^{2} M_{K}^{2}\left[\frac{7}{2}-\frac{3}{2} \ln \frac{M_{K}^{2}}{M_{V}^{2}}+I_{2}\left(\frac{M_{K}^{2}}{M_{V}^{2}}\right)\right] \\
I_{2}(z) & =\int_{0}^{1}(1+x)\left\{\ln \left[x+(1-x)^{2} z\right]-\frac{x}{x+(1-x)^{2} z}\right\} d x .
\end{aligned}
$$

Note that $F_{\Gamma}$ is finite and therefore scale independent. Expanding in $1 / M_{V}^{2}$ leads in an analogous way as before to an expansion of the integral $I_{2}(z)$ around $z=0$,

$$
\begin{aligned}
I_{2}\left(\frac{M_{K}^{2}}{M_{V}^{2}}\right) & =I_{2}(0)+\bar{I}_{2}\left(\frac{M_{K}^{2}}{M_{V}^{2}}\right) \\
& =-2.75+0.95,
\end{aligned}
$$

where $\bar{I}_{2}(z)$ is defined similarly to $\bar{I}_{1}(z)$. The remainder decreases the approximate result $I_{2}(0)$ by about $35 \%$. Inserted in (56) we find

$$
\left[\frac{7}{2}-\frac{3}{2} \ln \frac{M_{K}^{2}}{M_{V}^{2}}+I_{2}(0)+\bar{I}_{2}\left(\frac{M_{K}^{2}}{M_{V}^{2}}\right)\right]=2.08+0.95,
$$


where again we have given the remainder separately. The change from the leading order term in the heavy mass expansion to the exact result is large, about $46 \%$.

Therefore we conclude that higher order corrections to the leading order term in the heavy mass expansion may be large, depending on the size of the expansion parameter. The ratio $M_{K}^{2} / M_{V}^{2}$ is the largest value that enters our calculations, leading to corrections of the order of $50 \%$ in the example above. 


\section{B Contributions to $K_{i}^{R}$}

Below we list the finite contributions to the $K_{i}^{R}$ in their most general form, i.e. without using the relations in Eq.(14) for the parameters in the resonance sector. For completeness we give the contributions to the leading order constant $C$ [6]. Instead of $C$ we indicate the dimensionless quantity $Z=C / F_{0}^{4}$. Vanishing $K_{i}^{R}$ are omitted from the list.

$\underline{\text { Vector Mesons }}$

$$
\begin{array}{ll}
\frac{F_{V}^{2}}{}: & \\
Z^{V}\left(\mu_{0}\right)=-\frac{3}{2} \frac{1}{16 \pi^{2}} \frac{F_{V}^{2}}{F_{0}^{4}} M_{V}^{2}\left(\ln \frac{M_{V}^{2}}{\mu_{0}^{2}}+2\right) \\
K_{1}^{F_{V}}\left(\mu_{0}\right)=-\frac{3}{16} \frac{1}{16 \pi^{2}} \frac{F_{V}^{2}}{F_{0}^{2}}\left(\ln \frac{M_{V}^{2}}{\mu_{0}^{2}}+\frac{7}{6}\right) \\
K_{2}^{F_{V}}\left(\mu_{0}\right)=K_{1}^{F_{V}}\left(\mu_{0}\right) & K_{3}^{F_{V}}\left(\mu_{0}\right)=-K_{1}^{F_{V}}\left(\mu_{0}\right) \\
K_{4}^{F_{V}}\left(\mu_{0}\right)=2 K_{2}^{F_{V}}\left(\mu_{0}\right) & K_{5}^{F_{V}}\left(\mu_{0}\right)=-3 K_{1}^{F_{V}}\left(\mu_{0}\right) \\
K_{6}^{F_{V}}\left(\mu_{0}\right)=-3 K_{2}^{F_{V}}\left(\mu_{0}\right) & K_{11}^{F_{V}}\left(\mu_{0}\right)=-K_{1}^{F_{V}}\left(\mu_{0}\right) \\
K_{12}^{F_{V}}\left(\mu_{0}\right)=2 K_{1}^{F_{V}}\left(\mu_{0}\right) & \\
K_{13}^{F_{V}}=\frac{3}{4} \frac{1}{16 \pi^{2}} \frac{F_{V}^{2}}{F_{0}^{2}} & K_{14}^{F_{V}}=\frac{1}{2} K_{13}^{F_{V}} .
\end{array}
$$

$\underline{F_{V} G_{V}}:$

$$
\begin{array}{rlrl}
K_{1}^{F_{V} G_{V}}\left(\mu_{0}\right) & =\frac{3}{4} \frac{1}{16 \pi^{2}} \frac{F_{V} G_{V}}{F_{0}^{2}}\left(\ln \frac{M_{V}^{2}}{\mu_{0}^{2}}+\frac{1}{6}\right) \\
K_{2}^{F_{V} G_{V}}\left(\mu_{0}\right) & =K_{1}^{F_{V} G_{V}}\left(\mu_{0}\right) & K_{3}^{F_{V} G_{V}}\left(\mu_{0}\right)=-K_{1}^{F_{V} G_{V}}\left(\mu_{0}\right) \\
K_{4}^{F_{V} G_{V}}\left(\mu_{0}\right)=2 K_{2}^{F_{V} G_{V}}\left(\mu_{0}\right) & K_{5}^{F_{V} G_{V}}\left(\mu_{0}\right)=-3 K_{1}^{F_{V} G_{V}}\left(\mu_{0}\right) \\
K_{6}^{F_{V} G_{V}}\left(\mu_{0}\right)=-3 K_{2}^{F_{V} G_{V}}\left(\mu_{0}\right) & K_{12}^{F_{V} G_{V}}\left(\mu_{0}\right)=K_{1}^{F_{V} G_{V}}\left(\mu_{0}\right) \\
K_{13}^{F_{V} G_{V}}\left(\mu_{0}\right)=-2 K_{1}^{F_{V} G_{V}}\left(\mu_{0}\right) & K_{14}^{F_{V} G_{V}}\left(\mu_{0}\right)=K_{1}^{F_{V} G_{V}}\left(\mu_{0}\right) .
\end{array}
$$




$$
\begin{array}{ll}
\frac{F_{V}^{2} G_{V}^{2}}{}: & \\
K_{1}^{F_{V}^{2} G_{V}^{2}}\left(\mu_{0}\right)=-\frac{3}{8} \frac{1}{16 \pi^{2}} \frac{F_{V}^{2} G_{V}^{2}}{F_{0}^{4}}\left(\ln \frac{M_{V}^{2}}{\mu_{0}^{2}}+\frac{7}{6}\right) \\
K_{2}^{F_{V}^{2} G_{V}^{2}}\left(\mu_{0}\right)=K_{1}^{F_{V}^{2} G_{V}^{2}}\left(\mu_{0}\right) & K_{3}^{F_{V}^{2} G_{V}^{2}}\left(\mu_{0}\right)=-K_{1}^{F_{V}^{2} G_{V}^{2}}\left(\mu_{0}\right) \\
K_{4}^{F_{V}^{2} G_{V}^{2}}\left(\mu_{0}\right)=2 K_{2}^{F_{V}^{2} G_{V}^{2}}\left(\mu_{0}\right) & K_{5}^{F_{V}^{2} G_{V}^{2}}\left(\mu_{0}\right)=-3 K_{1}^{F_{V}^{2} G_{V}^{2}}\left(\mu_{0}\right) \\
K_{6}^{F_{V}^{2} G_{V}^{2}}\left(\mu_{0}\right)=-3 K_{2}^{F_{V}^{2} G_{V}^{2}}\left(\mu_{0}\right) & K_{13}^{F_{V}^{2} G_{V}^{2}}\left(\mu_{0}\right)=-4 K_{1}^{F_{V}^{2} G_{V}^{2}}\left(\mu_{0}\right) \\
K_{14}^{F_{V}^{2} G_{V}^{2}}\left(\mu_{0}\right)=2 K_{1}^{F_{V}^{2} G_{V}^{2}}\left(\mu_{0}\right) . &
\end{array}
$$

$$
\begin{array}{ll}
\underline{G_{V}^{2}}: \\
K_{1}^{G_{V}}\left(\mu_{0}\right)=\frac{3}{8} \frac{1}{16 \pi^{2}} \frac{G_{V}^{2}}{F_{0}^{2}}\left(\ln \frac{M_{V}^{2}}{\mu_{0}^{2}}+\frac{1}{6}\right) \\
K_{2}^{G_{V}}\left(\mu_{0}\right)=-K_{1}^{G_{V}}\left(\mu_{0}\right) & K_{3}^{G_{V}}\left(\mu_{0}\right)=-K_{1}^{G_{V}}\left(\mu_{0}\right) \\
K_{4}^{G_{V}}\left(\mu_{0}\right)=2 K_{2}^{G_{V}}\left(\mu_{0}\right) & K_{5}^{G_{V}}\left(\mu_{0}\right)=-3 K_{1}^{G_{V}}\left(\mu_{0}\right) \\
K_{6}^{G_{V}}\left(\mu_{0}\right)=-3 K_{2}^{G_{V}}\left(\mu_{0}\right) . &
\end{array}
$$

Using the relations $F_{V} G_{V}=F_{0}^{2}$ and $F_{V}=2 G_{V}$ (see Eq.(14)) the sum of the above expressions simplify to

$$
\begin{array}{ll}
Z^{V}\left(\mu_{0}\right)=-\frac{3}{16 \pi^{2}} \frac{1}{F_{0}^{2}} M_{V}^{2}\left(\ln \frac{M_{V}^{2}}{\mu_{0}^{2}}+2\right) & K_{1}^{V}\left(\mu_{0}\right)=\frac{3}{16} \frac{1}{16 \pi^{2}}\left(\ln \frac{M_{V}^{2}}{\mu_{0}^{2}}-\frac{23}{6}\right) \\
K_{2}^{V}\left(\mu_{0}\right)=-\frac{3}{16} \frac{1}{16 \pi^{2}}\left(\ln \frac{M_{V}^{2}}{\mu_{0}^{2}}+\frac{25}{6}\right) & K_{3}^{V}\left(\mu_{0}\right)=-K_{1}^{V}\left(\mu_{0}\right) \\
K_{4}^{V}\left(\mu_{0}\right)=2 K_{2}^{V}\left(\mu_{0}\right) & K_{5}^{V}\left(\mu_{0}\right)=-3 K_{1}^{V}\left(\mu_{0}\right) \\
K_{6}^{V}\left(\mu_{0}\right)=-3 K_{2}^{V}\left(\mu_{0}\right) & K_{11}^{V}\left(\mu_{0}\right)=\frac{3}{8} \frac{1}{16 \pi^{2}}\left(\ln \frac{M_{V}^{2}}{\mu_{0}^{2}}+\frac{7}{6}\right) \\
K_{12}^{V}=-\frac{3}{4} \frac{1}{16 \pi^{2}} & K_{13}^{V}=-4 K_{12}^{V} .
\end{array}
$$

Note that the contributions to $K_{14}^{V}$ cancel in the sum.

\section{$\underline{\text { Axial vector Mesons }}$}




$$
\begin{array}{ll}
Z^{A}\left(\mu_{0}\right)=\frac{3}{2} \frac{1}{16 \pi^{2}} \frac{F_{A}^{2}}{F_{0}^{4}} M_{A}^{2}\left(\ln \frac{M_{A}^{2}}{\mu_{0}^{2}}+2\right) \\
K_{1}^{A}\left(\mu_{0}\right)=-\frac{3}{16} \frac{1}{16 \pi^{2}} \frac{F_{A}^{2}}{F_{0}^{2}}\left(\ln \frac{M_{A}^{2}}{\mu_{0}^{2}}+\frac{7}{6}\right) \\
K_{2}^{A}\left(\mu_{0}\right)=-K_{1}^{A}\left(\mu_{0}\right) & K_{3}^{A}\left(\mu_{0}\right)=-K_{1}^{A}\left(\mu_{0}\right) \\
K_{4}^{A}\left(\mu_{0}\right)=2 K_{2}^{A}\left(\mu_{0}\right) & K_{5}^{A}\left(\mu_{0}\right)=-3 K_{1}^{A}\left(\mu_{0}\right) \\
K_{6}^{A}\left(\mu_{0}\right)=-3 K_{2}^{A}\left(\mu_{0}\right) & K_{11}^{A}\left(\mu_{0}\right)=K_{1}^{A}\left(\mu_{0}\right) \\
K_{12}^{A}\left(\mu_{0}\right)=2 K_{1}^{A}\left(\mu_{0}\right) & \\
K_{13}^{A}=-\frac{3}{4} \frac{1}{16 \pi^{2}} \frac{F_{A}^{2}}{F_{0}^{2}} & K_{14}^{A}=-\frac{1}{2} K_{13}^{A} .
\end{array}
$$

With the help of the two Weinberg sum rules (see Eq.(14) ) the sum of the expressions from the vector resonances in (63) and from the axial vector resonances in (64) reads

$$
\begin{array}{ll}
Z^{V+A}=\frac{3}{16 \pi^{2}} \frac{1}{F_{0}^{2}} M_{V}^{2} \ln 2 & \\
K_{1}^{V+A}=-\frac{3}{16} \frac{1}{16 \pi^{2}}(5+\ln 2) & K_{2}^{V+A}=-\frac{3}{16} \frac{1}{16 \pi^{2}}(3-\ln 2) \\
K_{3}^{V+A}=-K_{1}^{V+A} & K_{4}^{V+A}=2 K_{2}^{V+A} \\
K_{5}^{V+A}=-3 K_{1}^{V+A} & K_{6}^{V+A}=-3 K_{2}^{V+A} \\
K_{11}^{V+A}\left(\mu_{0}\right)=\frac{3}{16} \frac{1}{16 \pi^{2}}\left(\ln \frac{M_{V}^{2}}{\mu_{0}^{2}}+\frac{7}{6}-\ln 2\right) & \\
K_{12}^{V+A}\left(\mu_{0}\right)=-\frac{3}{8} \frac{1}{16 \pi^{2}}\left(\ln \frac{M_{V}^{2}}{\mu_{0}^{2}}+\frac{19}{6}+\ln 2\right) & \\
K_{13}^{V+A}=\frac{9}{4} \frac{1}{16 \pi^{2}} & K_{14}^{V+A}=\frac{1}{6} K_{13}^{V+A} .
\end{array}
$$

Therefore only $K_{11}^{V+A}\left(\mu_{0}\right)$ and $K_{12}^{V+A}\left(\mu_{0}\right)$ are scale dependent.

$\underline{\text { Scalar Mesons }}$ 
Octet :

$$
\begin{array}{ll}
K_{1}^{S}\left(\mu_{0}\right)=\frac{3}{4} \frac{1}{16 \pi^{2}} \frac{c_{d}^{2}}{F_{0}^{2}}\left(\ln \frac{M_{S}^{2}}{\mu_{0}^{2}}+\frac{1}{6}\right) \\
K_{2}^{S}\left(\mu_{0}\right)=-K_{1}^{S}\left(\mu_{0}\right) & K_{3}^{S}\left(\mu_{0}\right)=-\frac{1}{3} K_{1}^{S}\left(\mu_{0}\right) \\
K_{4}^{S}\left(\mu_{0}\right)=-\frac{2}{3} K_{1}^{S}\left(\mu_{0}\right) & K_{5}^{S}\left(\mu_{0}\right)=-K_{1}^{S}\left(\mu_{0}\right) \\
K_{6}^{S}\left(\mu_{0}\right)=K_{1}^{S}\left(\mu_{0}\right) .
\end{array}
$$

Singlet :

$$
\begin{aligned}
& K_{3}^{S_{1}}\left(\mu_{0}\right)=-\frac{3}{2} \frac{1}{16 \pi^{2}} \frac{\tilde{c}_{d}^{2}}{F_{0}^{2}}\left(\ln \frac{M_{S_{1}}^{2}}{\mu_{0}^{2}}+\frac{1}{6}\right) \\
& K_{4}^{S_{1}}\left(\mu_{0}\right)=2 K_{3}^{S_{1}}\left(\mu_{0}\right) .
\end{aligned}
$$

The numerical results at the scale point $\mu_{0}=M_{\rho}$ (with the values for the parameters given in Eqs.(15,17)) are presented in Table 3. There are no contributions to $K_{7} \ldots K_{10}$ from the resonances at all. For the nonvanishing couplings we find that the contributions from the vector and axial vector resonances dominate the $K_{i}^{R}$, like in the strong sector [6].

In Table 1 in Section 5 we list the algebraic expressions for $K_{1}^{R} \ldots K_{13}^{R}\left(K_{14}^{R}\right.$ does not contribute to physical amplitudes) using the relations in Eq.(14), also indicated are the numerical results at the scale point $\mu_{0}=M_{\rho}$.

\section{Large $N_{C}$}

In this appendix we classify the coupling constants $K_{i}$ according to their large $N_{C}$ behaviour. We then compare their behaviour to the numerical result obtained from the resonances.

In the large $N_{C}$ limit of QCD, operators that contain the product of two traces in flavour space are suppressed by $1 / N_{C}$ with respect to those operators with one flavour trace only [13. We apply this counting scheme to operators of order $O\left(e^{2} p^{2}\right)$ in the next-to-leading Lagrangian $\mathcal{L}_{4}^{Q}$. First of all we have to make two assumptions:

1. We assume that the electromagnetic interactions do not change the large $N_{C}$ counting of the strong force, i.e.

$$
M_{\pi}^{2} \sim O(1), \quad F_{\pi} \sim O\left(N_{C}^{1 / 2}\right) .
$$




\begin{tabular}{|l|r|r|r|r|r|}
\hline Type: & $V$ & $A$ & $S$ & $S_{1}$ & Total \\
\hline$Z^{R}\left(M_{\rho}\right)$ & -0.88 & 1.79 & 0 & 0 & $0.91^{(*)}$ \\
\hline \multicolumn{5}{|c|}{ Units: $\times 10^{-3}$} \\
\hline$K_{1}^{R}\left(M_{\rho}\right)$ & -4.6 & -2.2 & 0.4 & 0 & -6.4 \\
\hline$K_{2}^{R}\left(M_{\rho}\right)$ & -4.9 & 2.2 & -0.4 & 0 & -3.1 \\
\hline$K_{3}^{R}\left(M_{\rho}\right)$ & 4.6 & 2.2 & -0.1 & -0.2 & 6.4 \\
\hline$K_{4}^{R}\left(M_{\rho}\right)$ & -9.9 & 4.4 & -0.2 & -0.5 & -6.2 \\
\hline$K_{5}^{R}\left(M_{\rho}\right)$ & 13.7 & 6.6 & -0.4 & 0 & 19.9 \\
\hline$K_{6}^{R}\left(M_{\rho}\right)$ & 14.8 & -6.6 & 0.4 & 0 & 8.6 \\
\hline$K_{7}^{R} \ldots K_{10}^{R}$ & 0 & 0 & 0 & 0 & 0 \\
\hline$K_{11}^{R}\left(M_{\rho}\right)$ & 2.8 & -2.2 & 0 & 0 & 0.6 \\
\hline$K_{12}^{R}\left(M_{\rho}\right)$ & -4.7 & -4.4 & 0 & 0 & -9.2 \\
\hline$K_{13}^{R}$ & $19.0^{(*)}$ & $-4.7^{* *}$ & 0 & 0 & $14.2^{* *}$ \\
\hline$K_{14}^{R}$ & 0 & $2.4^{(*)}$ & 0 & 0 & $2.4^{(*)}$ \\
\hline
\end{tabular}

Table 3: Contributions from vector $(V)$, axial vector $(A)$, scalar octet $(S)$ and scalar singlet $\left(S_{1}\right)$ resonances to the couplings $Z$ and $K_{1} \ldots K_{14}$ at the scale point $\mu_{0}=M_{V} \simeq M_{\rho}$. We have used the relations in Eq.(14) and the numerical values for the parameters given in Eqs.(15,17). Scale independent quantities different from zero are signed with an asterisk ${ }^{(*)}$. 
From this we deduce

$$
C \sim O\left(N_{C}\right), \quad K_{i} \sim O(1), \quad i=1 \ldots 14
$$

where we made use of Eq.(4) and of the expressions for the masses [9, 10, for the $P_{\ell 2}$ decay constants [23] and for the vector and axial vector two point functions.

2. In the construction of $\mathcal{L}^{C}$ and $\mathcal{L}_{4}^{Q}$ local spurions $Q_{R}(x), Q_{L}(x)$ have been used which at the end are identified with the charge matrix $Q$ [6]. Therefore we consider $Q$ as a (spurious) source term and assume that the operator $\left\langle Q^{2}\right\rangle$ corresponds to a closed quark loop with a photon loop attached that interacts via gluons to other (flavourneutral) operators [34] .

Therefore the following parts of the Lagrangian $\mathcal{L}_{4}^{Q}$ could be suppressed,

$$
\begin{aligned}
& K_{1} F_{0}^{2}\left\langle d^{\mu} U^{\dagger} d_{\mu} U\right\rangle\left\langle Q^{2}\right\rangle, \quad K_{2} F_{0}^{2}\left\langle d^{\mu} U^{\dagger} d_{\mu} U\right\rangle\left\langle Q U Q U^{\dagger}\right\rangle, \\
& K_{3} F_{0}^{2}\left(\left\langle d^{\mu} U^{\dagger} Q U\right\rangle\left\langle d_{\mu} U^{\dagger} Q U\right\rangle+\left\langle d^{\mu} U Q U^{\dagger}\right\rangle\left\langle d_{\mu} U Q U^{\dagger}\right\rangle\right), \\
& K_{4} F_{0}^{2}\left\langle d^{\mu} U^{\dagger} Q U\right\rangle\left\langle d_{\mu} U Q U^{\dagger}\right\rangle, \\
& K_{7} F_{0}^{2}\left\langle\chi^{\dagger} U+U^{\dagger} \chi\right\rangle\left\langle Q^{2}\right\rangle, \quad K_{8} F_{0}^{2}\left\langle\chi^{\dagger} U+U^{\dagger} \chi\right\rangle\left\langle Q U Q U^{\dagger}\right\rangle .
\end{aligned}
$$

However, in the derivation of $\mathcal{L}_{4}^{Q}$ we have used trace identities that relate operators with different numbers of flavour traces [9], which may invalidate the suppression of the single operators listed above. Explicitly,

$$
\begin{aligned}
& \left\langle U^{\dagger} d_{\mu} U Q U^{\dagger} d^{\mu} U Q+U d_{\mu} U^{\dagger} Q U d^{\mu} U^{\dagger} Q\right\rangle= \\
& 2\left\langle\left\{d^{\mu} U^{\dagger}, d_{\mu} U\right\} Q^{2}\right\rangle-\left\langle d^{\mu} U^{\dagger} d_{\mu} U\right\rangle\left\langle Q^{2}\right\rangle \\
& +\left(\left\langle d^{\mu} U^{\dagger} Q U\right\rangle\left\langle d_{\mu} U^{\dagger} Q U\right\rangle+\left\langle d^{\mu} U Q U^{\dagger}\right\rangle\left\langle d_{\mu} U Q U^{\dagger}\right\rangle\right) \\
& \left\langle d_{\mu} U^{\dagger} Q d^{\mu} U Q\right\rangle= \\
& \left\langle d^{\mu} U^{\dagger} Q U\right\rangle\left\langle d_{\mu} U Q U^{\dagger}\right\rangle+\frac{1}{2}\left\langle d^{\mu} U^{\dagger} d_{\mu} U\right\rangle\left\langle Q U Q U^{\dagger}\right\rangle \\
& -\left\langle d^{\mu} U^{\dagger} d_{\mu} U Q U^{\dagger} Q U+d^{\mu} U d_{\mu} U^{\dagger} Q U Q U^{\dagger}\right\rangle
\end{aligned}
$$

Taking altogether, we find the following behaviour,

$$
\begin{aligned}
K_{i} & \sim O(1), \quad i=1 \ldots 14 ; i \neq 7,8 \\
K_{1}+K_{3}, 2 K_{2}-K_{4}, K_{7}, K_{8} & \sim O\left(1 / N_{C}\right) .
\end{aligned}
$$

In comparison to the couplings $L_{i}$ in the strong sector the $K_{i}$ are in general suppressed by a factor $1 / N_{C}$ due to the additional factor $F_{0}^{2}$ split off in the electromagnetic counterterms (see Eq.(6) ) and due to the first assumption made above. 
The suppression of $K_{8}$ implies that the contributions from the counterterms of order $O\left(e^{2} m_{s}\right)$ to $M_{\pi^{ \pm}}^{2}$ are small,

$$
\begin{aligned}
M_{\pi^{ \pm}}^{2}= & M_{\pi}^{2}+2 e^{2} \frac{C}{F_{0}^{2}}+e^{2} M_{K}^{2}\left\{8 K_{8}^{r}(\mu)-\frac{C}{F_{0}^{4}}\left[\frac{1}{8 \pi^{2}} \ln \frac{M_{K}^{2}}{\mu^{2}}+32 L_{4}^{r}(\mu)\right]\right\} \\
& +O\left(e^{2} M_{\pi}^{2}\right) .
\end{aligned}
$$

where $M_{\pi}, M_{K}$ are the leading order masses at $O\left(m_{q}\right)$ and the strong coupling constant $L_{4}$ is suppressed itself in the large $N_{C}$ limit [2].

The contributions from the resonances to the couplings $K_{i}^{R}$ vary over a large range, see Appendix B. We look out for the numerical contributions to the four quantities that are suppressed by $1 / N_{C}$. $K_{7}$ and $K_{8}$ are untouched by the resonances, whereas $K_{1}^{R} \ldots K_{4}^{R}$ get rather large resonance contributions. The relevant combinations, however, vanishes in nice agreement with the large $N_{C}$ limit,

$$
K_{1}^{R}\left(\mu_{0}\right)+K_{3}^{R}\left(\mu_{0}\right)=2 K_{2}^{R}\left(\mu_{0}\right)-K_{4}^{R}\left(\mu_{0}\right)=0 .
$$

\section{References}

[1] S.Weinberg, Physica A 96 (1979) 327.

[2] J.Gasser and H.Leutwyler, Ann. Phys. (NY) 158 (1984) 142; Nucl. Phys. B 250 (1985) 465.

[3] H.Leutwyler, Ann. Phys. (NY) 235 (1994) 165.

[4] For reviews of CHPT see:

H.Leutwyler, in: Proc. XXVI Int. Conf. on High Energy Physics, Dallas, 1992, Ed. J.R.Sanford, AIP Conf. Proc. No. 272 (AIP, New York, 1993) p.185;

in: Chiral Dynamics Workshop, Cambridge (MA), 1994, Eds. A.M.Bernstein and B.R.Holstein, Lecture Notes in Physics 452 (Springer, 1995) p.14;

U.-G.Meißner, Rep. Prog. Phys. 56 (1993) 903;

J.Bijnens, G.Ecker and J.Gasser, in: The Second Daథne Physics Handbook, Eds. L.Maiani, G.Pancheri and N.Paver (SIS, Frascati, 1995) p.125;

G.Ecker, Prog. Part. Nucl. Phys. 35 (1995) 1; Chiral Perturbation Theory, UWThPh-1996-34 (hep-ph/9608226);

A.Pich, Rep. Prog. Phys. 58 (1995) 563;

B.R.Holstein, Chiral Perturbation Theory: A Primer (hep-ph/9510344).

[5] G.Ecker, in: Chiral Dynamics Workshop, Cambridge (MA), 1994, Eds. A.M.Bernstein and B.R.Holstein, Lecture Notes in Physics 452 (Springer, 1995) p.41.

[6] G.Ecker, J.Gasser, A.Pich and E.de Rafael, Nucl. Phys. B 321 (1989) 311;

G.Ecker, J.Gasser, H.Leutwyler, A.Pich and E.de Rafael, Phys. Lett. B 223 (1989) 425. 
[7] T.Das, G.S.Guralnik, V.S.Mathur, F.E.Low and J.E.Young, Phys. Rev. Lett. 18 (1967) 759.

[8] W.A.Bardeen, J.Bijnens and J.-M.Gérard, Phys. Rev. Lett. 62 (1989) 1343.

[9] R.Urech, Nucl. Phys. B 433 (1995) 234.

[10] H.Neufeld and H.Rupertsberger, Z. Phys. C 68 (1995) 91.

[11] R.Baur, J.Kambor and D.Wyler, Nucl. Phys. B 460 (1996) 127.

[12] R.Baur and R.Urech, Phys. Rev. D 53 (1996) 6552.

[13] G.'t Hooft, Nucl. Phys. B 72 (1974) 461;

E.Witten, Nucl. Phys. B 160 (1979) 57.

[14] R. Dashen, Phys. Rev. 183 (1969) 1245.

[15] The different results for the corrections to Dashen's theorem are reviewed in: H.Leutwyler, Phys. Lett. B 378 (1996) 313;

G.Ecker, Chiral Perturbation Theory, UWThPh-1996-34 (hep-ph/9608226).

[16] D.Toublan, Phys. Rev. D 53 (1996) 6602.

[17] J.F.Donoghue, C.Ramirez and G.Valencia, Phys. Rev. D 39 (1989) 1947.

[18] S.Weinberg, Phys. Rev. Lett. 18 (1967) 507.

[19] E.Jenkins, A.Manohar and M.B.Wise, Phys. Rev. Lett. 75 (1995) 2272.

[20] E.G.Floratos, S.Narison and E.de Rafael, Nucl. Phys. B 155 (1979) 115;

P.Pascual and E.de Rafael, Z. Phys. C 12 (1982) 127.

[21] K.Huber and H.Neufeld, Phys. Lett. B 357 (1995) 221.

[22] A.Bramon, Phys. Lett. B 333 (1994) 153.

[23] H.Neufeld and H.Rupertsberger, Z. Phys. C 71 (1996) 131.

[24] R.Baur, Ph.D. Thesis, University of Zürich, 1996.

[25] A.Manohar and H.Georgi, Nucl. Phys. B 234 (1984) 189.

[26] J.Bijnens, Phys. Lett. B 306 (1993) 343.

[27] J.Gasser and H.Leutwyler, Phys. Rep. 87 (1982) 77.

[28] J.F.Donoghue, B.R.Holstein and D.Wyler, Phys. Rev. D 47 (1993) 2089.

[29] S.G.Gorishny, Nucl. Phys. B 319 (1989) 633. 
[30] S.G.Gorishny and S.A.Larin, Nucl. Phys. B 283 (1987) 452.

[31] G.B.Pivovarov and F.V.Tkachov, Int. Journ. Mod. Phys. A 8 (1993) 2241.

[32] V.A.Smirnov, Commun. Math. Phys. 134 (1990) 109; Renormalization and Asymptotic Expansion (Birkhäuser, Basel, 1991).

[33] V.A.Smirnov, Mod. Phys. Lett. A 10 (1995) 1485.

[34] D.Wyler, private communication.

[35] J.Bijnens and J.Prades, Electromagnetic Corrections for Pions and Kaons: Masses and Polarizabilites, FTUV/96-69 (hep-ph/9610360).

[36] W.A.Bardeen, A.J.Buras and J.-M.Gérard, Nucl. Phys. B293 (1987) 787.

[37] D.-N.Gao, M.-L.Yan and B.-A.Li, Electromagnetic mass splittings of $\pi, a_{1}$, $K, K_{1}(1400)$ and $K^{*}(892)$, NSC/USTC-29/96 (hep-ph/9611297).

[38] J.F.Donoghue and A.F.Pérez, The Electromagnetic Mass Differences of Pions and Kaons, UCTP-9-96 (hep-ph/9611331).

[39] W.N. Cottingham, Ann. Phys. 25 (1963) 424. 